\title{
Spontaneous emission in micro- or nanophotonic structures
}

\author{
Zhiyuan Qian' ${ }^{1}$, Lingxiao Shan' ${ }^{1}$, Xinchen Zhang ${ }^{1}$, Qi Liu' ${ }^{1,4}$, Yun Ma ${ }^{1}$, Qihuang Gong 1,2,3,4,5 \\ and Ying $\mathrm{Gu}^{1,2,3,4,5^{*}}$ (D)
}

*Correspondence: ygu@pku.edu.cn
'State Key Laboratory for
Mesoscopic Physics, Department of
Physics, Peking University, Beijing,
China
2Frontiers Science Center for
Nano-optoelectronics, Collaborative
Innovation Center of Quantum
Matter, and Beijing Academy of
Quantum Information Sciences,
Peking University, Beijing, China
Full list of author information is
available at the end of the article

\begin{abstract}
Single-photon source in micro- or nanoscale is the basic building block of on-chip quantum information and scalable quantum network. Enhanced spontaneous emission based on cavity quantum electrodynamics (CQED) is one of the key principles of realizing single-photon sources fabricated by micro- or nanophotonic cavities. Here we mainly review the spontaneous emission of single emitters in micro- or nanostructures, such as whispering gallery microcavities, photonic crystals, plasmon nanostructures, metamaterials, and their hybrids. The researches have enriched light-matter interaction as well as made great influence in single-photon source, photonic circuit, and on-chip quantum information.
\end{abstract}

Keywords: Spontaneous emission, Cavity quantum electrodynamics, Whispering gallery microcavities, Photonic crystals, Plasmon nanostructures, Metamaterials

\section{Introduction}

Single-photon source in micro- or nanoscale is the basic building block of on-chip quantum information and scalable quantum network. Enhancement of spontaneous emission (SE) in the frame of cavity quantum electrodynamics (CQED), i.e. Purcell effect controlling the spontaneous emission rate through cavity modes, is one of the key principles of realizing single-photon sources [1]. With the trend of on-chip optical quantum devices, realizing single photon emission in the micro- or nanoscale is increasingly important for quantum information process. Local field enhancement or small optical mode volume in micro- or nanostructures brings huge advantages in CQED, quantum information, and light-matter interaction. So far, the enhancement of SE in the micro- or nanoscale has reached great achievements in the areas of whispering gallery microcavities, photonic crystals (PCs), surface plasmon polaritons (SPPs), metamaterials, and so on, which provides abundant choices and methods of controlling and collecting single photons. In this Review, we will mainly focus on the theories, experiments, and up-to-date applications of $\mathrm{SE}$ in above areas. These researches demonstrate that Purcell effect has great influence in single-photon source, photonic circuit, and on-chip quantum information.

In the past decades, there were several excellent reviews related to the SE. For examples, Serge Haroche et al. used the concept of cavity quantum electrodynamics to review

(C) The Author(s). 2021 Open Access This article is licensed under a Creative Commons Attribution 4.0 International License which permits use, sharing, adaptation, distribution and reproduction in any medium or format, as long as you give appropriate credit to the original author(s) and the source, provide a link to the Creative Commons licence, and indicate if changes were made. The images or other third party material in this article are included in the article's Creative Commons licence, unless indicated otherwise in a credit line to the material. If material is not included in the article's Creative Commons licence and your intended use is not permitted by statutory regulation or exceeds the permitted use, you will need to obtain permission directly from the copyright holder. To view a copy of this licence, visit http://creativecommons.org/licenses/by/4.0/. 
the SE in 1989 [2], but not any photonic structure was involved. K. J. Vahala pointed out that optical microcavities confine light to small volumes by resonant recirculation which provides an environment for SE [3]. After that, towards to integrated quantum devices, there was a great achievement in this field. H. J. Kimble predicted that quantum nodes in quantum networks can be realized by the optical interactions of single photons and atoms in traditional CQED cavities [4]. In the last ten years, more and more researchers recognized the importance of quantum plasmonics and its applications in quantum information processing [5-7]. However, an updated version of reviewing the SE in microor nanophotonic structures is required, including not only the theoretical and practical knowledge accumulated over the past decades, but also the up-to-date applications and development trend of CQED system in the micro- or nanoscale, which is our intention of writing this review.

To quantitatively describe the enhancement of SE, Purcell factor $F$ is defined as $F=$ $\gamma_{t o t} / \gamma_{0}$, where $\gamma_{t o t}$ is the total SE rate from micro- or nanostructures and $\gamma_{0}$ in vacuum. According to Purcell theory, $F \propto Q / V$ with $V \sim L^{3}$, that is, larger Purcell enhancement requires larger quality factor $Q$ and smaller optical mode size $L$, which can be provided by various micro- or nanostructures. As shown in Table $1, Q$ and $L$ for traditional CQED systems are usually less than $10^{3}$ and $0.5 \mathrm{~mm}$, respectively, thus the Purcell factor $F$ is in the order of $10^{2}[2,3,8]$. With the larger $Q\left(10^{4} \sim 10^{9}\right)$ and smaller $L(10 \sim 100 \mu \mathrm{m})$, Purcell factors in whispering gallery microcavities can reach to $10^{5}$ [9-11]. By creating PC defect cavities, $L$ continues to get smaller to less than $10 \mu \mathrm{m}$, but $Q$ is limited in $10^{3} \sim 10^{5}$ due to the transport loss, so Purcell factors are usually less than $10^{2}[12-$ 15]. In SPP structures, the scale of $L$ can even be smaller than $1 \mu \mathrm{m}$ which is often in subwavelength scale and always leads to local field enhancement, but $Q$ is less than $10^{2}$ because of absorbtion loss, thus Purcell factors can be up to $10^{4}$ but with large absorption part [16-20]. Additionally, we also reviewed the photon emission in metamaterials and hybrid structures.

The rest of this Review is organized as follows: to ensure the completeness of the paper, in "A brief review of CQED" section, we briefly introduced the concept and developments of traditional CQED; Then in "The development of spontaneous emission in micro- or nanostructures" section, we summarized the theories, experiments, and applications of the spontaneous emission in various micro- or nanostructures, mainly including whispering gallery microcavities, photonic crystals, plasmon nanostructures, metamaterials,

Table 1 Schematic, mode size $L$, quality factor $Q$, and Purcell factors $F=\gamma_{\text {tot }} / \gamma_{0}$ for traditional CQED systems [2, 3, 8], WGMs [9-11], PCs [12-15], and SPP structures [16-20]

\begin{tabular}{|c|c|c|c|c|}
\hline Cavity & Tradition & WGM & PC & SPP \\
\hline \multicolumn{5}{|l|}{ Schematic } \\
\hline Mode size $L$ & $<0.5 \mathrm{~mm}$ & $10 \sim 100 \mu \mathrm{m}$ & $<10 \mu \mathrm{m}$ & $<1 \mu \mathrm{m}$ \\
\hline Quality factor $Q$ & $10^{3}$ & $10^{4} \sim 10^{9}$ & $10^{3} \sim 10^{5}$ & $<10^{2}$ \\
\hline $\begin{array}{l}\text { Purcell factor } \\
F=\gamma_{t o t} / \gamma_{0}\end{array}$ & $0 \sim 10^{2}$ & $10 \sim 10^{5}$ & $0 \sim 10^{2}$ & $0 \sim 10^{4}$ \\
\hline
\end{tabular}


and their hybrid structures; Finally, in "Summary and prospects" section, there were the summary and prospects.

\section{A brief review of CQED}

Cavity quantum electrodynamics focuses on the interaction between quantized field and quantum emitter at a single-quantum level in the limited space as well as cavities. The study of CQED started from Purcell's discovery in 1946 [1] that the spontaneous emission can be enhanced or inhibited by the surrounding environment. In 1963, Jaynes and Cummings $[21,22]$ set up the interaction between single-mode electromagnetic field and two-level atom, which is now known as Jaynes-Cummings model. In 1983, Goy's group used Rydberg $\mathrm{Na}$ atoms to increase the rate of spontaneous emission by about 50 times [23]. Later, Hulet's group achieved suppressed spontaneous emission of Rydberg Cs atoms experimentally [24]. At the end of 1980s, the theory of weak coupling regime of cavity quantum electrodynamics had been in-depth researched [2]. Owing to the improvement of micro- or nanocavity technology [25], strong interactions of single atoms and photons in CQED attracted much attention from quantum entanglement and cold atoms [26-30], which was decades later than weak coupling because of its rigorous conditions. In 1996, Serge Haroche finished the first proof of interaction between single two-level atom and single photon in the cavity [31], and thus became a Nobel laureate in 2012 [32]. Since then, strong and weak coupling regimes have been both key components of CQED [3, 4, 33-35].

Light trapped in the cavity can only form resonance at some specific eigenfrequencies, i.e., discrete cavity modes, leading to interesting behavior between cavity and emitter $[2$, 35-42]. Using Jaynes-Cummings model [21, 22] with single-mode cavity and two-level emitter [Figs. 1(a-f)], the Hamiltonian and dynamical equations are described as [43, 44]

$$
\begin{array}{r}
H=\hbar \omega_{a} \sigma^{\dagger} \sigma+\hbar \omega_{c} a^{\dagger} a+\hbar g\left(\sigma^{\dagger} a+\sigma a^{\dagger}\right), \\
\dot{\rho}=-(i / \hbar)[H, \rho]-(\gamma / 2)\left(\sigma^{\dagger} \sigma \rho-\sigma \rho \sigma^{\dagger}+H . c .\right) \\
-(\kappa / 2)\left(a^{\dagger} a \rho-a \rho a^{\dagger}+H . c .\right) .
\end{array}
$$

where $\sigma^{\dagger}(\sigma)$ is the rising (lowering) operator of the emitter, $a^{\dagger}(a)$ is the creation (annihilation) operator of the mode of nanocavity, and $\omega_{a}\left(\omega_{c}\right)$ is the frequency of the emitter (nanocavity). This CQED system is governed by three photonic processes $[5,7,20,26$, $44,45]$, characterized as parameters $g, \kappa$, and $\gamma$, where $g$ donates the coupling coefficient between emitter and cavity mode, $\kappa$ describes the dissipative channel from cavity to the thermal reservoir, and $\gamma$ describes the dissipative channel from emitter to the modes other than nanocavity mode. Two typical regimes are distinguished as weak coupling regime $(g \ll \gamma, \kappa)$ [Figs. 1(c, d)] and strong coupling regime $(g \gg \gamma, \kappa)$ [Figs. 1(c, e)].

When talking about weak coupling regime, because $g \ll \gamma, \kappa$, the energy exchanging strength between quantum emitter and cavity is much less than the loss rates of system, so most photons decay into the environment. The SE rate of quantum systems can be controlled by cavity mode and other modes out of cavity, which is so-called Purcell effect. Its emission enhancement is defined as Purcell factor $[3,5,7]$

$$
F=\frac{\gamma}{\gamma_{0}}=\frac{3}{4 \pi^{2}}\left(\frac{\lambda}{n}\right)^{3} \frac{Q}{V}
$$




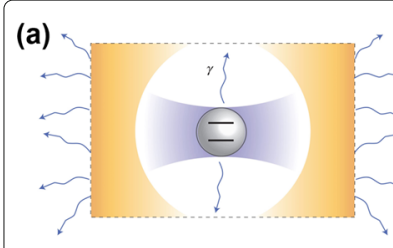

(d)

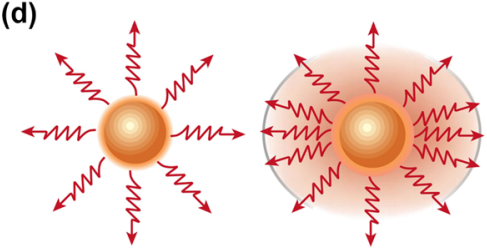

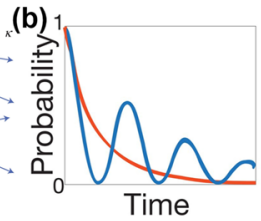

(e)

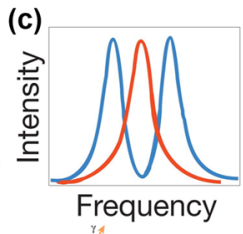

Frequency

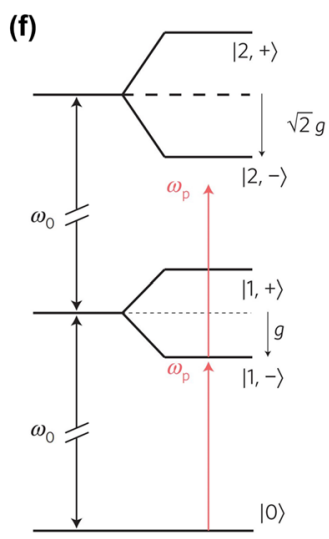

Fig. 1 Schematic diagram of CQED systems. a A typical CQED system consisting of a single quantum emitter (here is two level) and a cavity with coupling coefficient $g$, cavity loss $\kappa$, and spontaneous emission rate $\gamma$ [7]. b The probability of excited-state with time evolution and c transmission spectrum for weak (red curve) and strong (blue curve) coupling regimes [5]. d Schematic diagram of Purcell enhancement at weak coupling regime [3]. e Vacuum Rabi oscillation with period energy exchange at strong coupling regime [3]. f Schematic of Rabi splitting energy by dressed state theory [7]

which is proportional to quality factors $Q$ and inverse proportional to cavity mode volume $V$. For any resonant optical cavity, the $Q$ factor is defined as $Q=2 \pi$ (energy stored)/(energy lost in one cycle of oscillation) [46], thus $Q$ is inversely proportional to $\kappa$, which is much important for enhancement of SE in micro- or nanophotonic devices. Also, considering cavities may be open, cavity mode volume $V$ is an effective volume. Micro- or nanophotonic structures with small mode volume and high $Q$ could lead to huge enhancement of SE of quantum emitter due to $F \propto Q / V[5,6] . \gamma_{0}$ represents the spontaneous emission in vacuum, which can be obtained from Weisskopf-Wigner theory [47]:

$$
\gamma_{0}=\frac{1}{4 \pi \epsilon_{0}} \frac{4 \omega_{a}^{3} \mu^{2}}{3 \hbar c^{3}}
$$

where $\mu$ is the dipole moment of quantum emitter.

While for strong coupling regime, i.e., $g \gg \kappa, \gamma$, the energy exchanging strength between quantum emitter and cavity mode is much larger than the loss rate of the system. The coupling coefficient $g$ is described as

$$
g=\frac{\vec{E} \cdot \vec{\mu}}{\hbar}=\mu \sqrt{\frac{\omega_{c}}{2 \hbar \varepsilon V}}
$$

where $\vec{E}$ is the electric field corresponding to a single excitation and $\varepsilon$ means the environmental permittivity. The coupling coefficient $g \propto Q / \sqrt{V}[5,7]$. The Rabi splitting caused by strong coupling can be explained by the dressed state theory [48] and in the resonant condition it is

$$
E_{R}=2 \sqrt{g^{2}-(\kappa-\gamma)^{2} / 16}
$$

If $g$ is much larger than $|(\kappa-\gamma) / 4|$, Rabi splitting is close to $2 g$ [Fig. 1(f)].

Spontaneous emission of quantum emitters of CQED is quite important for realizing single photon sources. Rydberg atoms [22, 49,50] and quantum dots [51] are two kinds of technologic realization of single-photon sources owing to the large dipole moments. Also, quantum dots are popularly applied in Purcell experiments with different functionalities 
caused by various ingredients [52-55]. For example, InAs quantum dot embedding in GaAs materials can provide broadband spontaneous emission control with high efficiency [56-58], while entangled photon pair sources show high brightness and indistinguishability by combining GaAs quantum dots with new broadband photonic nanostructures [59].

The large enhancement of spontaneous emission makes CQED systems elemental in the field of quantum network, which plays an important role in the broad context of quantum information [4]. In the localized quantum nodes, the quantum state is generated, processed and stored in the setting of CQED. And quantum states are transported through quantum channels from site to site with high fidelity. Such quantum networks can be achieved by single photons and atoms provided by CQED systems [4, 36, 60, 61].

\section{The development of spontaneous emission in micro- or nanostructures}

According to Purcell's theory, the SE of quantum emitters is controlled by the optical modes of the cavity [1]. To engineer the optical modes in the micro- or nanoscale, people fabricate various micro- or nanostructures, such as nanofiber [62, 63], metal-coated nanostructure [64, 65], erbium dopants in a cryogenic resonator [66], cold atoms in microcavity [27-30], and so on. Some of them possess unique nature, like superradiance for atoms [67] and quantum state controlled emission [68]. Nowadays, traditional CQED study starts to combine with micro- or nanophotonics. Here we mainly focus on the spontaneous emission in whispering gallery microcavities, PCs, plasmonic structures, metamaterials, and hybrid photonic structures.

\section{Spontaneous emission in whispering gallery microcavities}

Whispering gallery modes (WGMs) are optical modes in which light is confined by continuous total internal reflection in microcavities with circular symmetry [Figs. 2(a, b)] [69-72]. The study of WGMs started from the research of acoustic wave propagation phenomena in the dome of St Paul's Cathedral in 1910 [73], where one could always hear the whisper of the others at anywhere of the gallery if they all stood near the wall. Since then, electromagnetic WGM microcavities with various shapes were found [74], such as spheres, pillars, disks, and toroids [69]. For a typical 2D WGM microcavity, light propagates in a circular pattern along the cavity wall, so only those modes satisfying $m \lambda=n_{e} L$ can be self-reinforced, while others are eliminated [Figs. 2(c, d)]. Here, $m$ is an integer angular mode number, $\lambda$ is the vacuum wavelength, $n_{e}$ is the effective refractive index, and $L$ is the circumference of the microcavity. Some characteristics of WGMs have also been observed, like radiation pressure enhancement effect [75] and structure resonances [76]. The first WGM laser was realized in 1961 [77], and then low-threshold version was developed for integrated optical chip in 1991 [78].

Compared with traditional Fabry-Perot cavities, WGM microcavities have higher quality factor $Q$, smaller mode volume $V$, longer photon lifetime, and larger optical density [69, 71, 79-82]. For example, WGMs of silica microsphere provide $Q$ of $\sim 10^{9}$ and small mode volume [79]. Normally, quality factors $Q$ of microcavities are not affected by emitters. However, in cases of emitters with large size, emitters' permittivity makes influence in optical mode of cavities, which leads to the change of quality factors $Q[69,83]$. According to Eq. (3), higher quality factor $Q$ and smaller mode volume $V$ lead to larger Purcell factor. Therefore, enhanced spontaneous emission can be easily realized in WGM cavities 
(a)

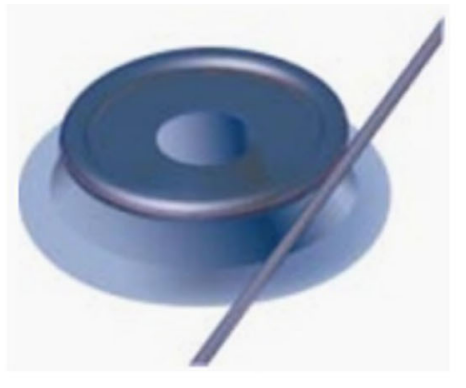

(c)

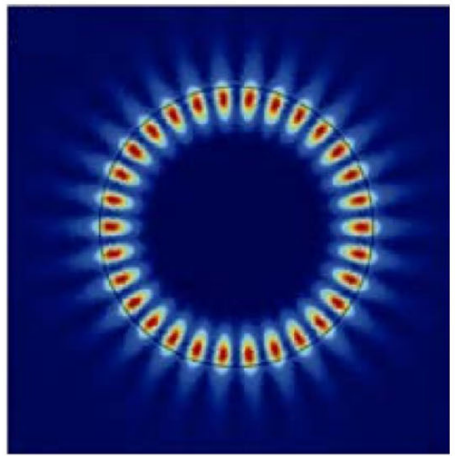

(b)

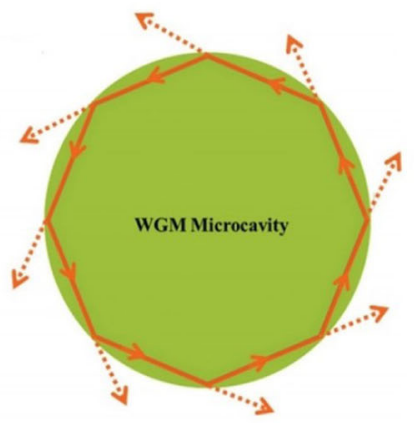

(d)

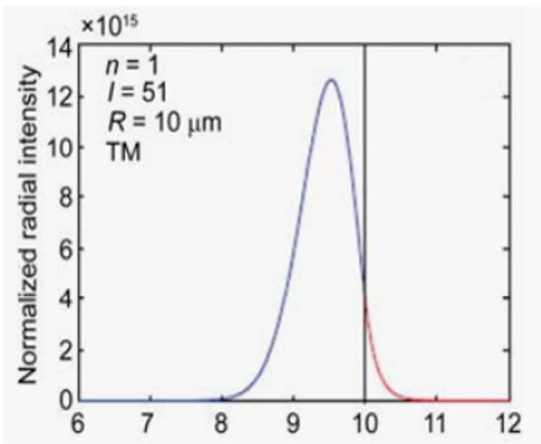

Fig. 2 The basic concepts of WGMs. a Schematic of WGM toroid microcavity [3]. $\mathbf{b}$ Schematic of self-reinforced light by total internal reflection [69]. c The typical electric field distribution of WGM in a microsphere [70]. d The radial field distribution of mode shown in (c) [70]. The $x$-axis in (d) represents radius distance from center, and the unit is $\mu \mathrm{m}$

$[10,80]$. For the applications in single photon sources [3, 84-88], we concentrate on several typical structures consisting of WGMs and quantum emitters [3, 10, 11, 89-93].

The first experiment of spontaneous emission of single quantum dot in microdisk was realized in 2000, with the Purcell factor about 6 [10, 84] [Figs. 3(a, b)]. Owing to the strong temperature dependence of single-exciton resonance of the quantum dot, energy from quantum dot to WGMs could be controlled. Same phenomenon was also demonstrated in microsphere cavity [89] [Figs. 3(c, d)]. Spontaneous emission suppression was later demonstrated in similar structures using metal sidewall coatings [90]. In order to measure the spontaneous emission of WGM structures, in 2003, Vahala's group proposed fiber-taper coupling to high- $Q$ microspheres [92]. Using a combination of lithography, dry etching and a selective reflow process, they achieved Purcell factor to $10^{5}$ in on-chip silica toroid-shaped microresonators [Fig. 3(e)] with ultrahigh $Q / V$ [Fig. 3(f)] [11, 91]. In addition, strong coupling behavior in WGM cavities has also been well developed, where periodical energy exchanging between WGM and emitter leads to Rabi splitting [94-97].

Single photon source is one of the most important applications of the Purcell effect in microcavities [98]. The pioneering work of single photon source was done in 2000 by A. Kiraz et al. [84]. By using WGM cavity with the zero-phonon line of nitrogen-vacancy (NV) center, Purcell factor of 12 could be reached, with $25 \%$ zero-phonon line emission of diamond [88]. Therefore, by enhancing spontaneous emission and collecting single photons, WGM microcavities can realize an efficient integrable single photon source. 
(a)

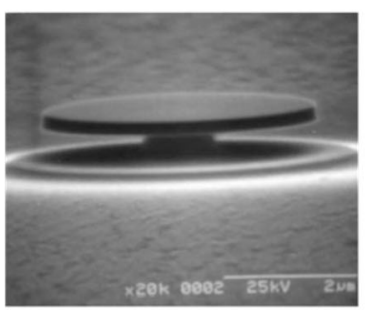

(c)

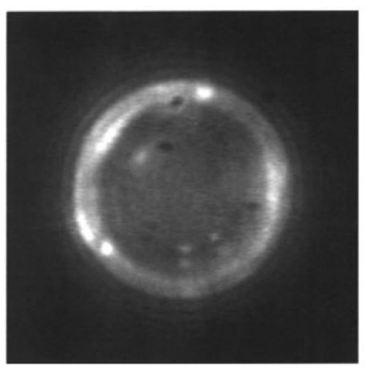

(e)

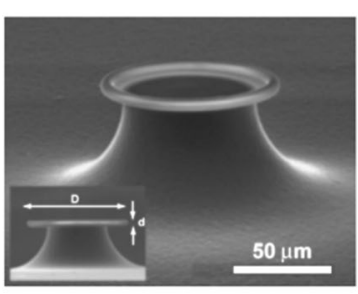

(d)
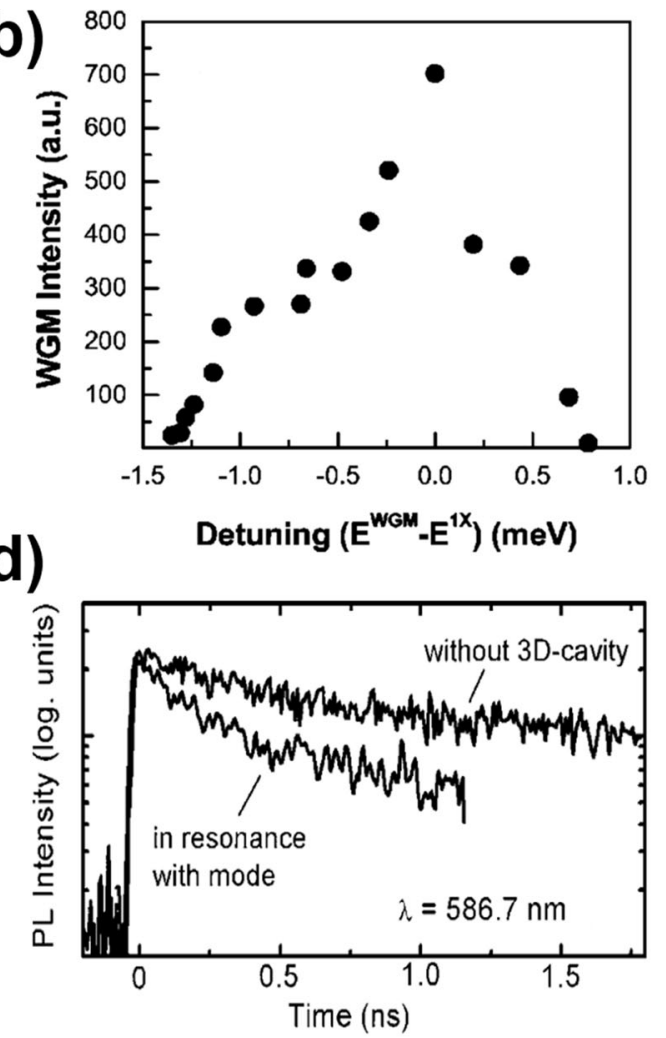

(b)

(f)

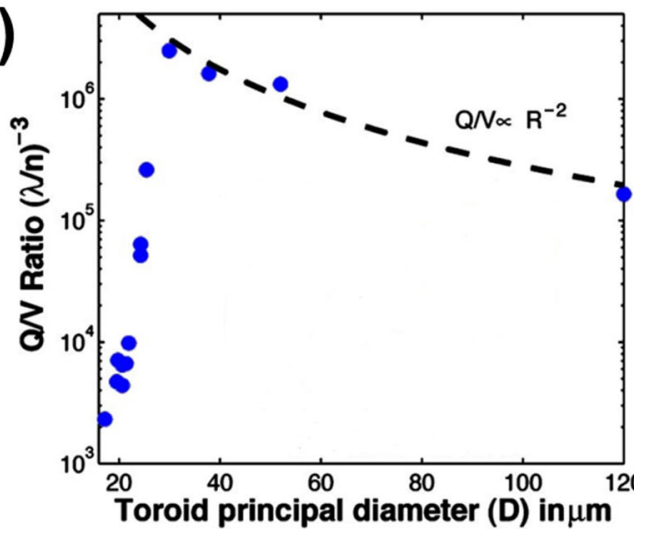

Fig. 3 SE based on WGM structures. a The microdisk structure containing a single InAs quantum dot [84]. b Enhancement of spontaneous emission when WGM and exciton are on resonance [10]. c A glass microsphere doped with CdSe nanocrystals in a thin surface shell [89]. d The radiative lifetime of CdSe quantum dots with and without WGM structure [89]. e A high Q/V toroid microcavity on a chip [11]. f Measured $Q / V$ ratio of toroid microcavities by varying principal diameter $D[11]$

Besides single photon sources, spontaneous emission of WGMs has also been applied in single-photon transport [99], quantum many-body simulation [100], and quantum gate [101].

\section{Spontaneous emission in photonic crystals (PCs)}

\section{Introduction to $P C s$}

Photonic crystals impose periodic modulation on the refractive index in the scale of wavelengths [Figs. 4(a, b)], allowing Bloch modes for photons similar with electrons in crystal lattices [106]. In the reciprocal space, Bloch modes form photonic band diagrams 
(a)

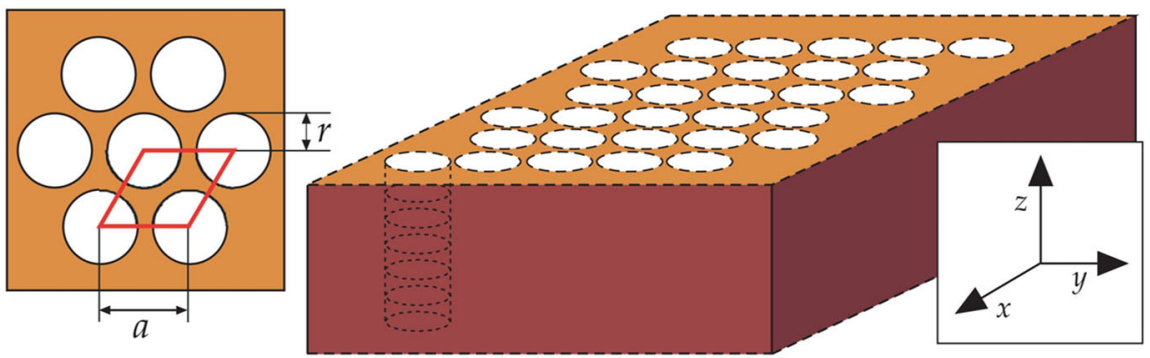

(b)

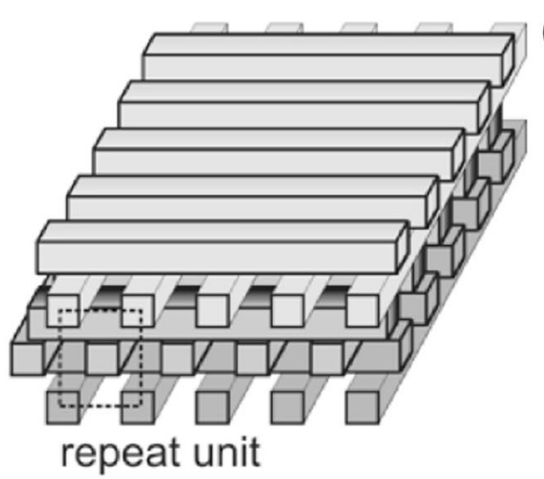

(c)

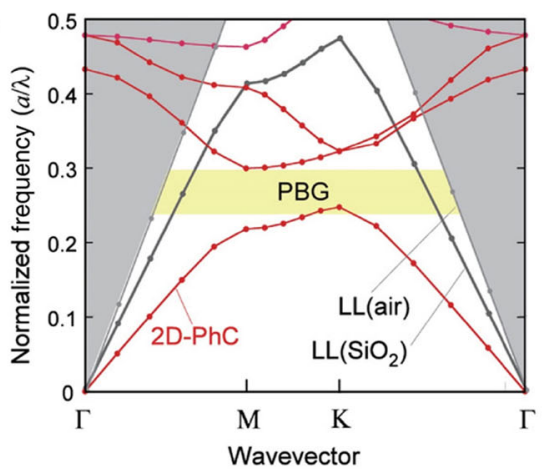

(e)

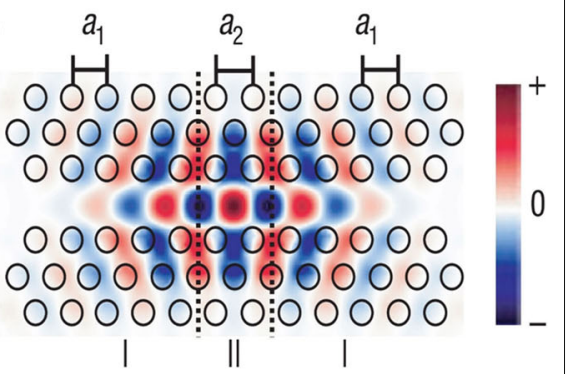

Fig. 4 The basic concepts of PCs. a A schematic diagram of a 2D PC slab [102]. b A schematic diagram of 3D PC [103]. c A photonic band diagram of a 2D PC slab [104]. d The electric field in a L3 cavity by shifting the holes at the cavity edges [12]. e The electric field of a double heterostructure PC waveguide [105]

while the frequency range without Bloch modes is called the photonic band gap (PBG) [Fig. 4(c)]. Thus, undesired radiation can be suppressed in the PBG by the deliberate design of PCs $[107,108]$. The ability of radiation suppression differs in various PC structures [102, 104]. Only the PBG in 3D PCs can be complete [103], where optical modes are prohibited in all wavevectors and polarizations. With easier fabrication for on-chip devices, 2D PCs show higher potentials in applications despite of incomplete band gap.

Defect modes in the PBG $[109,110]$ are commonly utilized as optical microcavities, which can be introduced by removing lattice units $[109,111]$ and changing size or refractive index [112]. These modes possess narrow linewidths due to the suppression of other undesired modes, where electric field is confined in the subwavelength scale [3]. PC microcavities usually possess $10^{3} \sim 10^{5}$ of $Q$-factors and moderate $V\left(\sim(\lambda / n)^{3}\right)$ $[12,112-119]$. The most common design of photonic crystal microcavity is L3 PC microcavity [Fig. 4(d)], which is produced by removing 3 holes along a row of hexagonal lattices and changing the geometry of several neighbouring holes. Such design avoids the abrupt change at the edge of the microcavity that undesired scattering can be suppressed 
[12, 120-122]. Another scheme of defect modes is to remove the entire row of hexagonal lattices, called W1 defect waveguide [123], which allows light propagation in the line defect. Quality factor $Q$ can be further improved by special modification of PC structures [13], especially in double-heterostructure nanocavity [Fig. 4(e)] with $Q \sim$ $6 \times 10^{5}[105]$. Thus, the PC microcavity with high $Q$ and small $V$ enables the enhancement of spontaneous emission.

PC structures can be fabricated by up-to-date nanotechnologies. Due to the difficulty of the 3D nanofabrication, 2D PCs have occupied the most attention [15, 124, 125]. To optimize the radiation effect in defect modes, the frequency and spatial position of emitters should be accurately determined. The frequency between cavity and emitters should match, which can be realized by tuning temperature [126], inert gas [127] and electric field [128]. The spatial position of emitters requires positioning technology [5, 129-131]. The DNA origami technology provides an effective way with accurate positioning of $10-\mathrm{nm}$ solution [132].

In recent years, some novel PCs with unique optical dispersion relationship have been developed, such as index-near-zero materials [133] and Dirac/Weyl-type PCs [134137], which are different from bandgap PCs. These PCs with new characteristics, like long-range dipole-dipole interactions $[135,136]$, may lead to more interesting results of spontaneous emission.

\section{Spontaneous emission in PCs}

When considering emitters in PC structures, PBGs and defect modes play an important role in modulating spontaneous emission [104]. With the state-of-art nanotechnology, various components have been introduced into PC structures for enhanced localized density of states (LDOS), such as metal [14, 138], silicon nitride [139], gallium phosphide [140], organic materials [141], and two-dimensional materials [142-144]. When emitter is on resonance with defect modes, the high LDOS results in the emission enhancement. Among 2D PC structures, defect square-lattices cavity [15] and PC waveguides [145] can support Purcell enhancement. For 3D PCs, spontaneous emission could be controlled in the infrared region $[146,147]$. While emission frequency falls in the photonic band gap, the density of states is lower than that in vacuum, leading to the suppressed emission. The emission could be suppressed in the 3D multilayer 'woodpile' structure [111] and enhanced in the 3D 'inverse woodpile' structure with two-fold Purcell factor [147]. Some characteristics of PC may help control spontaneous emission, such as slow light [148151], Fano resonance [152-158], and quasicrystals [159-161].

The Purcell enhancement can be realized easily in PC defect cavities. In the defect cavity of square lattices [15], Purcell factor reached 76 and the delay time after the pump in laser response was cut down to a few picoseconds. Thus the early work of single photon sources in PC was conducted in the inverse opal 3D PCs with 5-fold Purcell enhancement [Figs. 5(a, b)] [162]. The Purcell effect also provides possibilities for tailoring polarization by air holes of PC defects [164-166]. The single photon emission with linear polarization was achieved in the point defect by designing a PBG only for transverse-electric-like modes [125].

Compared to defect cavities, PC waveguides have the advantage in the high coupling efficiency $[163,167,168]$. Emitted photons can be coupled into the waveguide mode, especially one-way PC waveguide [Figs. 5(c, d)] supporting unidirectional light emission. 
(a)

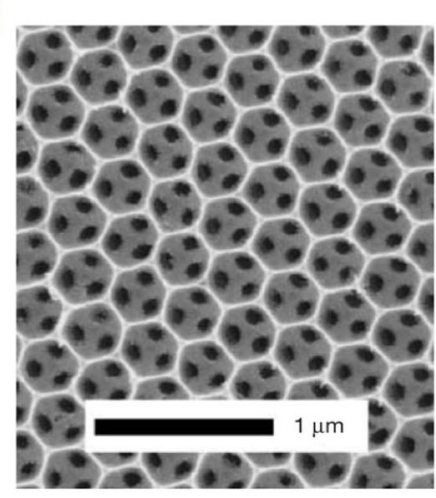

(c)

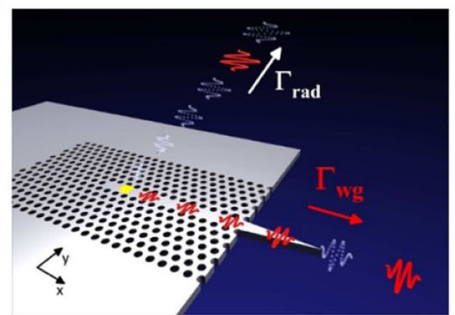

(b)

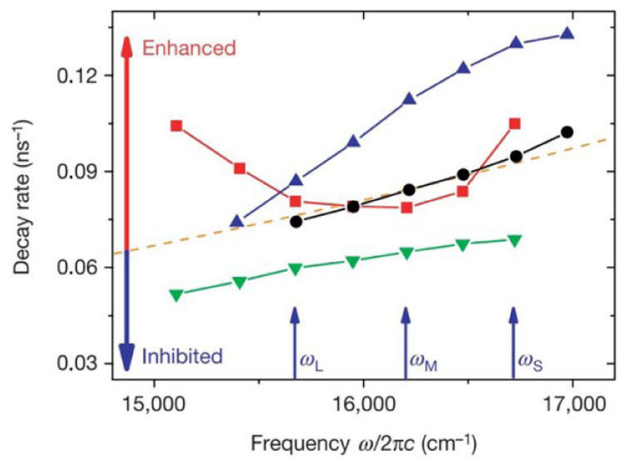

(d)

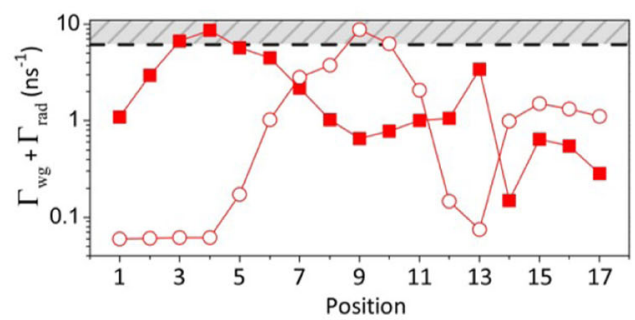

Fig. 5 Spontaneous emission enhancement in PC structures. a The SEM structure image of the inverse opal 3D PCs [162]. b Measured decay rates of the excited states in the inverse opal 3D PCs with different lattice parameters [162]. c Illustration of a single photon source based on a one-way PC waveguide [163]. d Effective decay rate with different positions in the structure (c) [163]

Also, the 'spin-moment locking' mechanism enables unidirectional transmission [169171]. The enhanced photons emitted from circularly polarized emitter can stimulate the specific transverse spin momentum to achieve the unidirectional transmission [148].

With the increase of $Q$ values of PC microcavities, the interaction between defect modes and the emitters goes into the strong coupling regime i.e., $g>\kappa, \gamma$ [12]. In the PC cavities with point defect, Rabi splitting has been observed [126, 129, 172]. Strongly coupled lightmatter systems provide anharmonic energy structure that allows photon blockade [7], which can be used in various PC microcavities for single photon sources [173-178].

\section{Spontaneous emission of single emitters in plasmon structures}

\section{Introduction to surface plasmon polaritons (SPPs)}

The history of surface plasmon polariton (SPP) can be traced back to 1900s [179-181]. Since then, mathematical description of the surface waves had been established and loss phenomena of metallic surfaces had been recorded [182-184]. The researches focusing on the trivial surface modes and related phenomenon were reviewed by $\mathrm{H}$. Raether et al [185]. Then the sandwich-like metallic structures with symmetric and asymmetric SPP modes were studied [186, 187]. Also, more localized field enhancement nearby various metallic nanoparticles was investigated in the micro- or nanoscale [17, 18, 188192]. As shown in Fig. 6, two classes of SPPs, i.e., localized SPPs supported by a metallic nanoparticle [Fig. 6(a)] and SPPs propagating along the interface [Fig. 6(d)] are introduced $[5,188]$. 
(a)

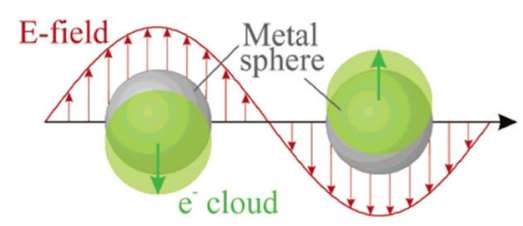

(c)

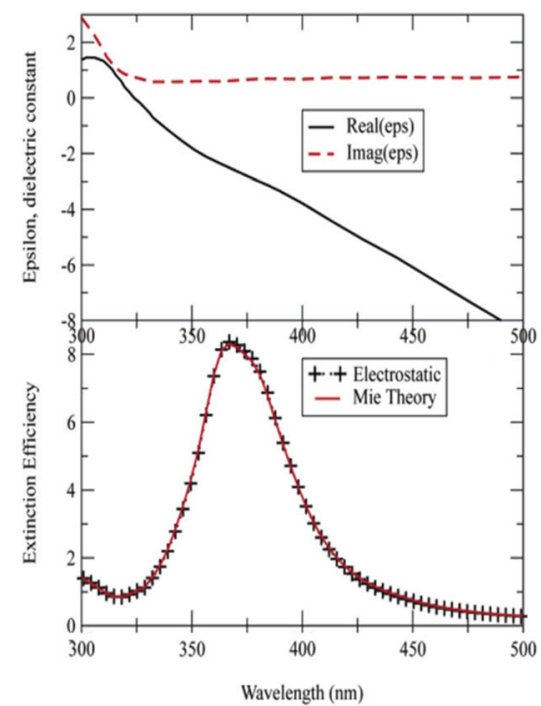

(b)

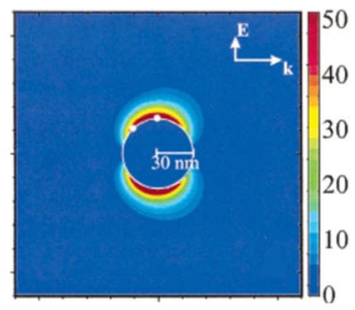

(d)

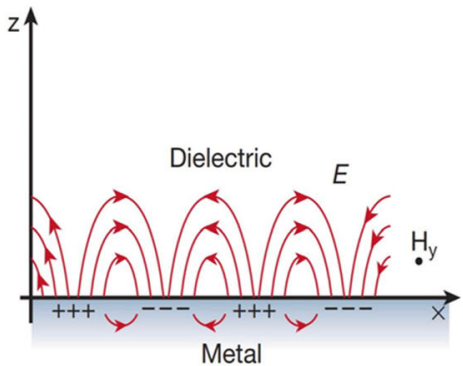

(e)

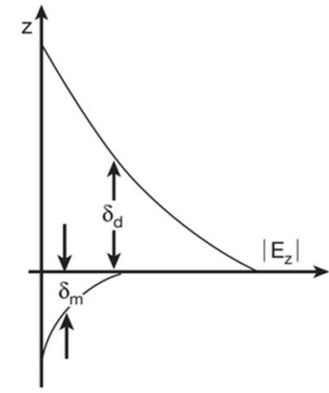

Fig. 6 SPP basics. a Schematic diagram of plasmon oscillation for a sphere [192]. b Electric field contours and c Extinction efficiency of dipole mode of a silver nanosphere with $r=30 \mathrm{~nm}$ [192]. d Schematic diagram of SPP wave at a metal-dielectric interface [17]. e The field decaying exponentially with $\delta_{m}$ in the metal and $\delta_{d}$ in the dielectric for SPP waves [17]

The localized SPPs of metallic spheres can be obtained from Mie's theory [188, 193, 194]. For a small enough metal sphere, i.e., $R \ll \lambda$, only dipole resonance is considered and its extinction cross section is described as $[193,194]$

$$
\sigma_{\text {ext }}(\omega)=9 \frac{\omega}{c} \epsilon_{d}^{3 / 2} V_{0} \frac{\epsilon_{m}^{\prime \prime}(\omega)}{\left[\epsilon_{m}^{\prime}(\omega)+2 \epsilon_{d}\right]^{2}+\epsilon_{m}^{\prime \prime}(\omega)^{2}},
$$

where $V_{0}=(4 \pi / 3) R^{3}$ denotes the particle volume, $\epsilon_{d}$ is the embedding dielectric permittivity, and $\epsilon_{m}(\omega)=\epsilon_{m}^{\prime}(\omega)+i \epsilon_{m}^{\prime \prime}(\omega)$ is the metal permittivity. The dipole resonance occurs at $\epsilon_{m}^{\prime}=-2 \epsilon_{d}$, which is independent on the radius of metallic sphere. Also, more higher-order resonances can appear when the metallic sphere becomes larger [194]. Besides nanosphere, other nanoparticles such as nanorods have the similar results [195]. When localized SPP happens, most electromagnetic energy concentrates near the metallic nanoparticle [5], namely, it has very small optical mode volume $V$ [Fig. 6(b)]. Though the quality factor $Q$ is not very high [Fig. 6(c)], it will enhance the light-matter interaction with the principles of CQED.

While for the SPP propagating along the metallic and dielectric surface, its dispersion relation is $[17,18,185,186,188]$ 


$$
k_{s p}=k_{0} \sqrt{\frac{\epsilon_{d} \epsilon_{m}}{\epsilon_{d}+\epsilon_{m}}},
$$

where $\epsilon_{d}$ and $\epsilon_{m}=\epsilon_{m}^{\prime}+i \epsilon_{m}^{\prime \prime}$ are the dielectric and metallic permittivity respectively. $k_{s p}$ is the complex surface plasmon wavevector, i.e., $k_{s p}=k_{s p}^{\prime}+i k_{s p}^{\prime \prime}$ with

$$
\begin{aligned}
& k_{s p}^{\prime}=k_{0} \sqrt{\frac{\epsilon_{d} \epsilon_{m}^{\prime}}{\epsilon_{d}+\epsilon_{m}^{\prime}}}, \\
& k_{s p}^{\prime \prime}=k_{0}\left(\frac{\epsilon_{d} \epsilon_{m}^{\prime}}{\epsilon_{d}+\epsilon_{m}^{\prime}}\right)^{3 / 2} \frac{\epsilon_{m}^{\prime \prime}}{2 \epsilon_{m}^{\prime 2}} .
\end{aligned}
$$

This dispersion relation shows that the light cannot couple with the SPP directly because $k_{s p}>k_{0}$ for the same frequency $\omega$ [17]. The $k_{z}$ in metal or dielectric medium is imaginary, so the field in this perpendicular direction is evanescent [Fig. 6(e)] as $z_{d}$ and $z_{m}$ respectively [185]

$$
\begin{aligned}
& z_{d}=\frac{\lambda}{2 \pi} \sqrt{\frac{\epsilon_{m}^{\prime}+\epsilon_{d}}{\epsilon_{d}^{2}}}, \\
& z_{m}=\frac{\lambda}{2 \pi} \sqrt{\frac{\epsilon_{m}^{\prime}+\epsilon_{d}}{\epsilon_{m}^{\prime 2}}} .
\end{aligned}
$$

For examples, when $\lambda=600 \mathrm{~nm}$, one obtains for silver [196] $z_{m}=23 \mathrm{~nm}$ and $z_{d}=371$ $\mathrm{nm}$, and for gold [196] $z_{m}=29 \mathrm{~nm}$ and $z_{d}=281 \mathrm{~nm}$. Thus SPP is localized at the interface between metal and dielectric and decays exponentially [189], which causes ultrasmall optical mode volume $V$ and can be applied to the spontaneous emission enhancement.

The propagation length of SPP can be found from the imaginary part of wavevector [17],

$$
\delta_{s p}=\frac{1}{2 k_{s p}^{\prime \prime}}=\frac{1}{k_{0}}\left(\frac{\epsilon_{m}^{\prime}+\epsilon_{d}}{\epsilon_{m}^{\prime} \epsilon_{d}}\right)^{3 / 2} \frac{\epsilon_{m}^{\prime}{ }^{2}}{\epsilon_{m}^{\prime \prime}} .
$$

For silver in visible spectrum, $\delta_{s p}$ ranges from 10 to $100 \mu \mathrm{m}$, and in near-infrared telecom spectrum $\delta_{s p}$ can reach $1 \mathrm{~mm}$ [17]. This propagation length is enough to support SPP waves in well-designed subwavelength photonic devices [190, 197]. Finally, as shown in Fig. 6(d), SPPs only exist for TM polarization [18, 191]. All above characteristics of SPP are based on single flat interface between a dielectric and metallic medium, but the same characteristics are also found in multilayer plasmon nanostructures $[18,187]$.

Modern nanofabrication technology especially chemical synthetic technology [198, 199], makes it possible to fabricate plasmonic structures. Localized SPPs have been proven by the synthesis of nanoparticles like nanobars, nanorice, and nanorods [198, 200, 201]. SPP waves in waveguides with various shapes such as nanoscale metallic cylindrical cable [202] and metal-insulator-metal structure [203] have also been studied. Hybrid integration of nanowires makes the propagation length of SPPs enough to function in the subwavelength photonics [197, 199, 204, 205]. Dark plasmons in metallic nanoparticles excited by localized emitter allows for subwavelength guiding of optical energy with almost no radiative losses [206]. The interaction between semiconductor and metallic nanoparticles has been studied, which brings unique nonlinear Fano effect [207, 208]. Theory of quantum optics with surface plasmons has also been constructed [209], which opens the way to the later studies of plasmon CQED and on-chip quantum information. 
Additionally, new types of lasers based on SPPs have been developed greatly, named as spasers [210].

SPPs have been widely applicated in plasmonic sensors [186, 211, 212], surfaceenhanced Raman scattering [213], and photothermal and magnetooptic effects [214, 215]. Electromagnetic field enhancement induced by SPPs makes quantum effects easier to observe and control on the chip, such as on-chip quantum interference [216], quantum entanglement [217], quantum dot integration [218], and quantum coherence enhancement [219].

\section{Spontaneous emission based on SPP structures}

Nowadays, plasmonic devices contribute a lot on strong and weak coupling systems of CQED by providing ultrasmall optical mode volume to enhance light-matter interaction [5-7, 220, 221]. According to localization properties of optical field, we roughly divided the studies of plasmonic CQED into three categories, i.e., localized SPP-based CQED, SPP-based CQED, and gap-plasmon-based CQED. The typical localized SPPbased CQED system [Fig. 7(a)] is composed of a single emitter like excited molecule or quantum dot, and a metal nanoparticle such as nanorod, nanosphere, or nanocube. The total decay rate $\gamma_{t o t}$ of a single emitter is obtained by considering two channels, one is the radiative loss $\gamma_{\text {rad }}$ emitting to the free space, the other is the nonradiative decay $\gamma_{a b s}$ originating from Ohmic loss inside the metal nanoparticle, i.e., $\gamma_{t o t}=\gamma_{\text {rad }}+\gamma_{a b s}$. Accordingly, the total, radiative, and nonradiative Purcell factors are obtained by $\gamma_{t o t} / \gamma_{0}, \gamma_{\text {rad }} / \gamma_{0}$ and $\gamma_{a b s} / \gamma_{0}$, respectively. $\gamma_{0}$ represents the spontaneous emission in vacuum. The total Purcell factor of localized SPP system usually ranges from $10^{0}$ to $10^{4}$ according to the distance between the emitter and plasmon structure [222].

The spontaneous emission of an excited molecule as emitter near a small metal sphere was calculated in the early 1980s [225]. The lifetime of an emitter near metal sphere decreases as emitter approaches the sphere because of nonradiative part $\gamma_{a b s}$. Also, oscillations in the lifetime occur even at separations of a few wavelengths, which means localized SPP is excited. So far, with the help of advanced microscope technology [Fig. 7(b)], the enhancement and quenching of emitter fluorescence coupling to noble metal nanoparticle have been investigated [195, 223, 226-228]. In practical researches, quantum yield $q_{a}=\gamma_{\text {rad }} / \gamma_{t o t}$ is the radio of radiative decay rate and represents the emission probability. The decrease of quantum yield means quenching of spontaneous emission. The local field enhancement of nanoparticle at localized SPP mode leads to an increased excitation rate whereas nonradiative energy transfer to the particle leads to a decrease of the quantum yield, which shows either fluorescence enhancement or quenching [Fig. 7(c)]. Moreover, the continuous transition from enhancement to quenching was demonstrated with tuning the distance between emitter and nanoparticle [223]. Besides nanospheres, other shapes of particles like nanorods [195], nanorice [198], and nanodisks [229] also provide spontaneous emission enhancement based on localized SPP. To obtain the Purcell factors near the plasmon structures, people have developed various methods, including semiclassical theory [222], Greenfunction [223, 230], first-principles [8], and the commercial softwares of FDTD and FEM [20].

In SPPs, evanescent waves decay exponentially perpendicular to the surface, which makes the electromagnetic modes compressed nearby the surface, thus enhances the 
(a)

\section{Metal Nanoparticle} (MNP)

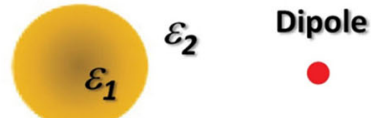

(b)

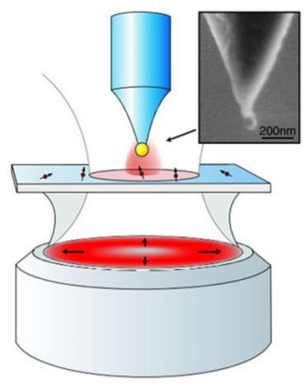

(c)

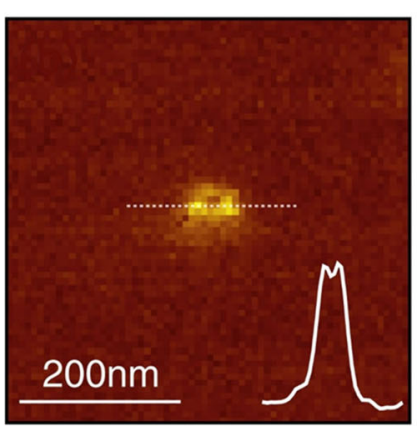

(d)

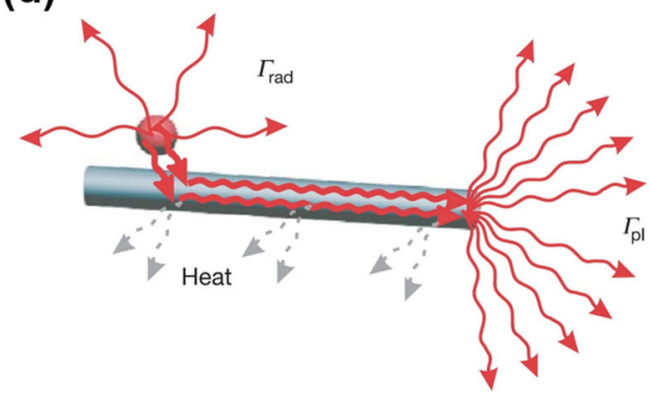

(e)

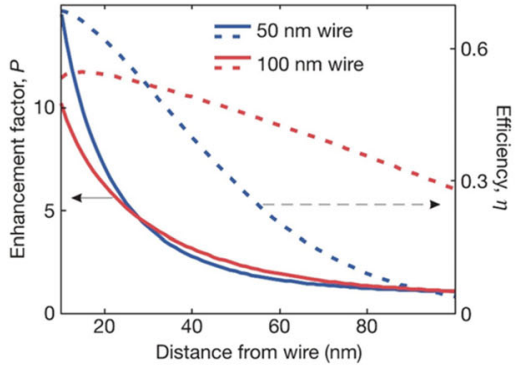

(f)

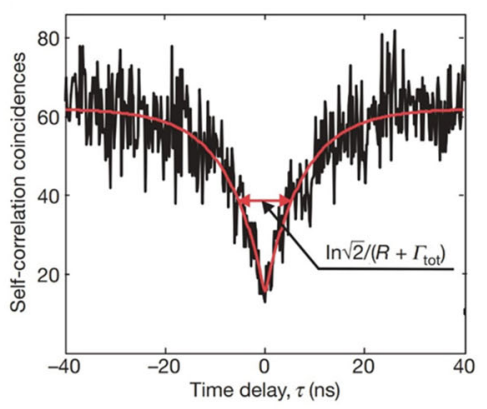

Fig. 7 Spontaneous emission based on SPP structures. a Schematic diagram of localized SPP-based CQED system [222]. b Sketch of the experimental arrangement with SEM image of a gold nanoparticle [223]. c Fluorescence image of particle-emitter interaction with dip in the center indicating quenching [223]. d Schematic diagram of SPP-based CQED system [224]. e Purcell factor (solid curve) and efficiency of emission into surface plasmons (dashed curve) with different size of wires [224]. f Second-order correlation function $g^{(2)}(\tau)$ of quantum dot fluorescence using SPP-CQED system [224]

light-matter interaction. The typical SPP-based CQED system [Fig. 7(d)] is usually composed of a single emitter and an one-dimension plasmonic nanowire or two-dimension plasmonic nanofilm. In this case, the total decay rates $\gamma_{t o t}$ includes three parts, first is the radiative loss $\gamma_{\text {rad }}$ emitting to the free space, second is effective part $\gamma_{e v}$ that guided into the SPP through the whole waveguide, third is the Ohmic loss $\gamma_{a b s}$ into the SPP waveguide. Accordingly, the total, radiative, nonradiative, and effective Purcell factors are obtained by $\gamma_{t o t} / \gamma_{0}, \gamma_{\text {rad }} / \gamma_{0}, \gamma_{a b s} / \gamma_{0}$, and $\gamma_{e v} / \gamma_{0}$, respectively. Purcell factor of these SPPbased CQED systems is around $10^{0} \sim 10^{1}[224,231,232]$, which is usually smaller than that of localized SPP-based CQED system.

In 2007, the research showed the generation of single plasmons in silver nanowires coupled to quantum dots [224]. When a single emitter is optically excited in proximity to a silver nanowire, emission photons couple directly to guided surface plasmons in 
the nanowire, causing the wire's end to light up [Fig. 7(d)]. The emitter-SPP coupling is stronger for thinner wires, while the out-coupling efficiency of SPP decreases for thinner wires [Fig. 7(e)]. Thus it's important to choose suitable size of nanowires to keep the balance between the decay rate and collection efficiency. By observing second-order correlation function $g^{(2)}(\tau)$ of quantum dot fluorescence [Fig. 7(f)], it's demonstrated that the light emission at the nanowire end is a single quantized surface plasmon [231, 232]. Considering the $2.5 \sim 10$ folds of Purcell enhancement [Fig. 7(e)], SPP-based CQED system at weak coupling regime is a good candidate for single-photon source. Meanwhile, the lifetime of exciton is shortened and the broadband nature of coupling is also demonstrated. Furthermore, the SPP waveguides can act as a planar dielectric antenna if multilayer (three layers or more) plasmon structures instead of simple nanowires or nanofilms are used. When placing emitters into their middle layer, the photon collection efficiency at the vertical direction almost approaches to $100 \%$ [233, 234], which can be used to realize a deterministic single-photon source. Therefore, with the advantage of efficiently collecting single photons, SPP-based CQED systems are quite useful in single-photon source, long-range optical coupling of quantum bits, and so on.

\section{Spontaneous emission based on gap SPP structures}

Gap plasmons exist in the nanoscale gap between two plasmon structures [Figs. 8(a, c, $e, h, j)]$. Thanks to the nanofabrication techniques [235], researchers have fabricated and investigated various plasmon structures with nanoscale gaps, such as double metal particles [16, 236-238], double SPP waveguides [239, 240], and metal particles with plasmonic waveguide [20, 45, 241-244]. In these structures, dramatic enhancement of SE is obtained due to more ultrasmall optical volume in the nanoscale gap.

The typical bowtie nanoantenna [Fig. 8(a)] is composed of two nearby metallic nanotriangles and a single emitter such as a molecule within the gap [16, 236]. An enhancement or quenching of the photon emission relies not only on the local-field factor and the quantum yield, but also on the excitation and emission spectra of the emitter [236]. Smaller gap of a metallic bowtie nanoantenna brings stronger local-field factor and less quantum yield. When a bowtie antenna is excited at the localized SPP frequency, light is focused to a hotspot within the gap, leading to a strong enhancement of the SE. Also, the bowtie antenna can function as a low-pass filter for emitter. In the experiment, by using gold bowtie and a single fluorescent molecule with low quantum efficiency, enhancement up to a factor of 1340 is observed [Fig. 8(b)], showing great potential for high-contrast selection of single nanoemitters [16]. Similarly, other nanoantennas of two metal particles like metal double-spheres [237] or metal double-rods [238] with nanoscale gap, also provide localized gap plasmons which can be applied for large enhancement of the SE.

Large SE enhancement can also occur in the nanogap between two plasmon waveguides, where the emitted photons can couple into guided SPPs. One example is the nonresonant enhancement of spontaneous emission in metal-dielectric-metal (MDM) slab waveguide structures [Fig. 8(c)] [240, 247]. Because of tight confinement of electric fields within the gap, these structures show the strong emission enhancement with factor of $10^{1} \sim 10^{2}$ [Fig. 8(d)] with almost $100 \%$ coupling efficiency to plasmon waveguides. Asymmetric gap waveguide, composed of a silver nanowire and a silver substrate separated by nanoscale dielectric bilayer of $\mathrm{Al}_{2} \mathrm{O}_{3}$ embedding the fluorescent dyes $\mathrm{Alq}_{3}$ [245], is also investigated [Fig. 8(e)]. This structure with strong field confinement within the 
(a)

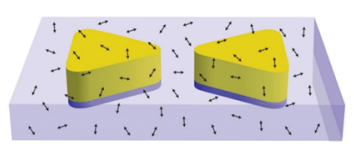

(c)

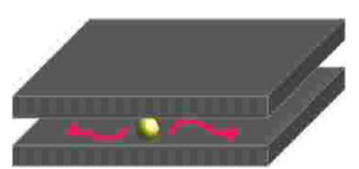

(e)

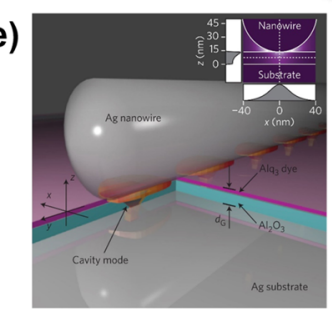

(b)

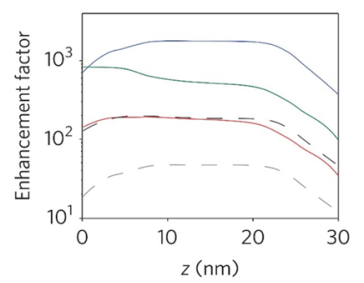

(d)

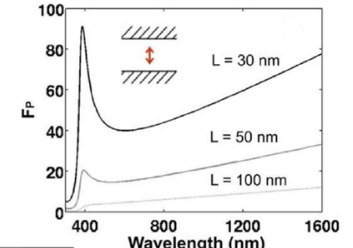

(f)

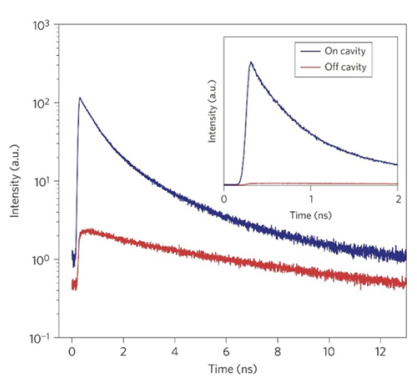

(h)

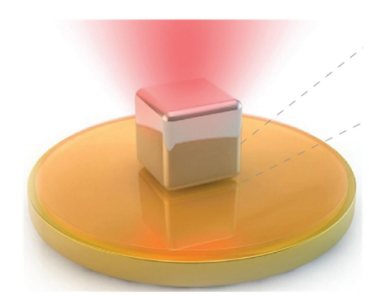

(j)

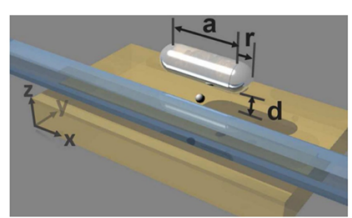

(g)

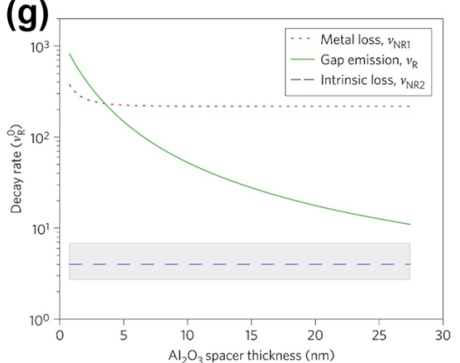

(i)

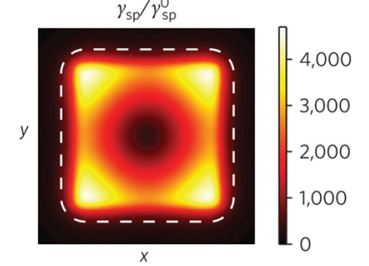

(k)

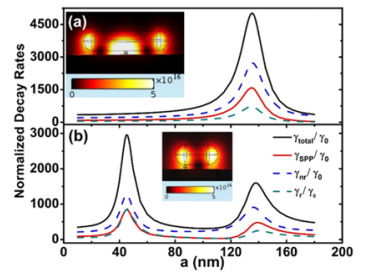

Fig. 8 SE enhancement based on gap SPP structures. a Schematic of gold bowtie nanoantenna with molecules (black arrows) as emitters [16]. b Radiative (red) and nonradiative (green) enhancement factors of structure (a) along the center of the gap [16]. c Schematic of metal-dielectric-metal (MDM) gap plasmon slab structure [240]. d Spontaneous emission enhancement for a MDM structure with different gap sizes [240]. e Schematic diagram of gap-plasmon-based CQED system [245]. f Time-resolved fluorescence measurements for the structure (e)[245]. $\mathbf{g}$ Decay rate for the structure (e) with different photonic channels [245]. $\mathbf{h}$ Schematic of a silver nanocube situated on a gold film [246]. i Distribution of enhancement of spontaneous emission rate for the structure (h) [246]. j Schematic diagram of silver nanorod-coupled gold nanofilm gap plasmon system with a designed nanofiber [20]. k Purcell factors for different emission paths for the structure (j) with emitter within the nano gap [20] 
gap can be seen as the nanocavity containing coupled emitters. The time evolution of the emission intensity is also greatly modified on or off resonance to the cavity [Fig. 8(f)]. As shown in Fig. 8(g), smaller gap brings stronger photon emission. The Purcell factor can even approach to $10^{3}$ when the emitters are placed within the nanogap [245].

Nanocavity consisting of metallic nanoparticles and plasmonic waveguide will be ultrasmall enough to explore more properties of CQED system including both weak and strong coupling regime due to the existence of the gap plasmon. Plenty of researches showed that these nanogaps structures can overcome the challenges of directional emission, room-temperature, broadband operation, high radiative quantum efficiency, and a large spontaneous emission rate [229, 246, 248-250]. By a film-coupled metal nanocube system with emitters embedded in the dielectric gap [Fig. 8(h)], SE enhancement can exceed $10^{3}$ [Fig. 8(i)] with high quantum efficiency (> 50\%) and collection efficiency (84\%) [246]. This work proves that smaller gap size brings stronger Purcell enhancement with maximum factor $\sim 2 \times 10^{3}$. Moreover, the longitudinal and transverse modes of film-coupled metal nanoparticle system are revealed to collect the emission photons [248]. For collecting and guiding the enhanced photons directly into the on-chip optical circuits in the nanoscale, metallic nanorod-coupled nanofilm structures coupled to a low-loss dielectric nanofiber are designed [Fig. 8(j)] [20]. Purcell factors of all these channels are shown in Fig. $8(\mathrm{k})$, with the order of magnitude $10^{3}$. Based on this design, by tuning the permittivity of the environment via liquid crystal, high-contrast switching of the spontaneous emission can be achieved, companying with high-efficiency extraction of the emitting photons [251]. To overcome high optical loss in the metal, some nanogaps between the dielectric and metallic structures are developed [252, 253]. These kind of devices with high quantum efficiency promise an important impact on various fundamental and applied research fields, including photophysics, ultrafast plasmonics, bright single-photon sources, and Raman spectroscopy.

\section{Plasmonic nanolasers \& strong coupling}

Plasmonic nanolaser is one of the key components for on-chip optical information systems based on SPP combining with simulated emission. Highly localized plasmonic mode allows lasers to work in the subwavelength scale [254-258]. Under the Purcell enhancement, lifetime of emitted photons is changed in well-designed nanocavity, enabling ultrafast responses of nanolasers [259]. In particular, spaser is one type of nanolasers, which can directly generate stimulated emission of quantized surface plasmons [7, 259]. The principle of spaser was proposed in 2003 [260], but the large absorption in metal prevented its realization. Until 2009, the first spaser-based nanolaser overcoming loss was demonstrated [261]. Soon the dark emission of spasers was revealed [262]. So far, ultralow-threshold plasmonic lasers under continuous-wave pumping at room temperature have been realized [263-265]. Overall, plasmonic nanolasers have great potential in circuits, sensors, superresolution imaging and quantum information, especially chemical and biological nanoscopic sensors [254, 259, 262, 266].

Surface plasmon combining with strong coupling regime of CQED also reaches great achievements. Different from cold-atom studies [26, 30], researchers can explore strong plasmon-exciton coupling based on plasmonics at room temperature [19, 267-274], which requires ultrasmall optical mode volume. In practice, it's hard to reach $g \gg \gamma, \kappa$, thus the strong coupling is usually confirmed by observing Rabi splitting of fluorescence 
spectrum and dynamics of reversible interaction [45, 275, 276]. Nowadays, strong coupling has been realized in several designs. By using special emitters such as $J$ aggregates, Rhodamine 6G molecules, or other quantum dots, hybrid plasmonic structures enable strong coupling [230, 267, 268, 271, 272, 274, 277-279]. Also, gap plasmon structures provide ultrasmall optical volume for strong coupling regime, such as dimer nanoantennas [269, 270] and hybrid nanowires coupling with metallic film [273]. Particularly, when considering evanescent mode from 1D or 2D waveguides [280, 281], the enhancement of light-matter interaction benefits from not only gap surface plasmon [19, 282], but also compressed optical mode volume by evanescent waves [45, 275, 276], leading to strong photon-exciton coupling with efficient fluorescence collection and high sensitivity to embedding environment.

\section{Spontaneous emission in metamaterials}

Metamaterials are artificial materials with distinctive optical characteristics including negative or zero refractive index [283-293]. The idea of metamaterial was firstly proposed by V. G. Veselago in 1968 [294], then realized by J. B. Pendry in 1999 [283, 284]. Owing to the unique structure designs, novel applications of metamaterials are provided, such as super-lenses, invisibility cloaks, trapped rainbow, optical blackhole, and superresolution imaging $[285,286,295,296]$. Nowadays, metamaterials have been developed into several branches such as negative index materials, zero index materials, and metasurfaces. Here we mainly introduce the spontaneous emission enhancement in metasurfaces and epsilon-near-zero (ENZ) materials.

\section{Spontaneous emission control with metasurfaces}

Metasurface can be seen as a 2D counterpart of metamaterials [297-300], which was firstly realized by using subwavelength optical antennas in 2011 [Fig. 9(a)] [301]. When light flows through metasurface, phase [Fig. 9(b)] [302], frequency [Fig. 9(c)] [303], and polarization [Fig. 9(d)] [304] of reflected and refracted light can be controlled by the designed metasurface. So far, people have made significant advances in metasurface, such as planar photonic devices [297], polarization conversion [298], radiation manipulation [306, 307], and spatiotemporal light control [308]. Recently, metasurface has been demonstrated as a suitable platform for quantum optics [309], including quantum coherence [310, 311], quantum interference [305, 312], and quantum entanglement [313].

In metasurface, Purcell effect mainly depends on the coupling interaction between emitters and metasurface [314-316]. The first research was made by using plasmonic metasurface in 2010, resulting in the Purcell factor of $10^{2}$ [317]. Later, metasurface made up of metal nanoparticles array was fabricated, exhibiting twofold Purcell enhancement in visible spectrum [318]. Purcell effect in metasurface was also realized by Yuan et al. [319] through designing all-dielectric metasurface composed of asymmetric geometric metaatoms. This structure with Purcell enhancement was also proven by other researchers $[320,321]$. Besides carefully designing metasurface, there are some other ways to enhance the photon emission in metasurface, such as constructing partially reflecting cavity [322] and changing the background refractive index by liquid crystals [323].

The metasurface's ability to modulate spontaneous emission brings novel applications in quantum interference. One fascinating example is anisotropic quantum vacuum [305] 


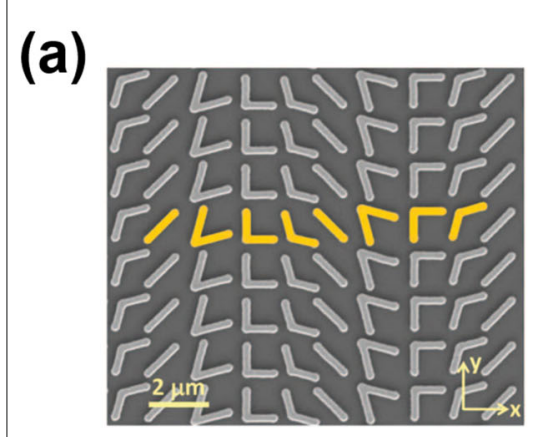

(c)

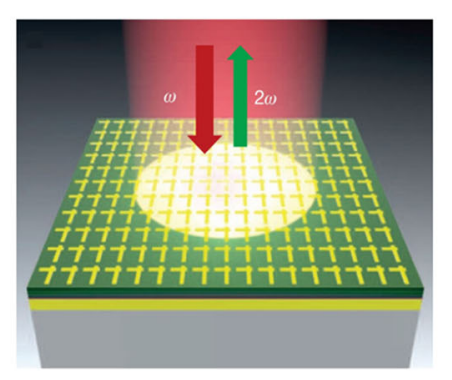

(e)

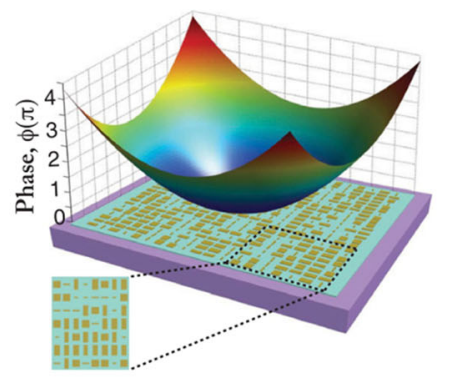

(b)

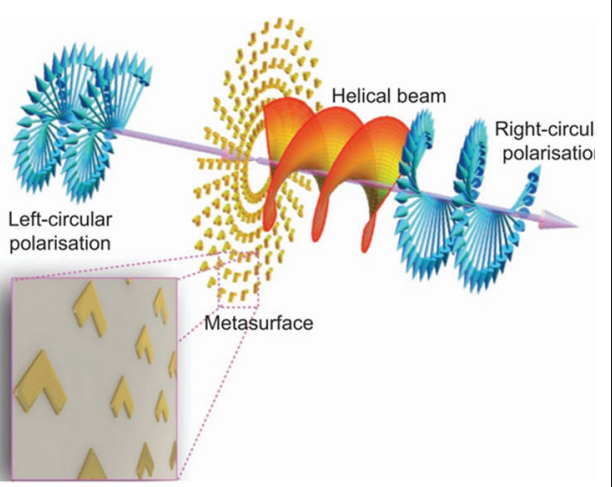

(d)

(f)
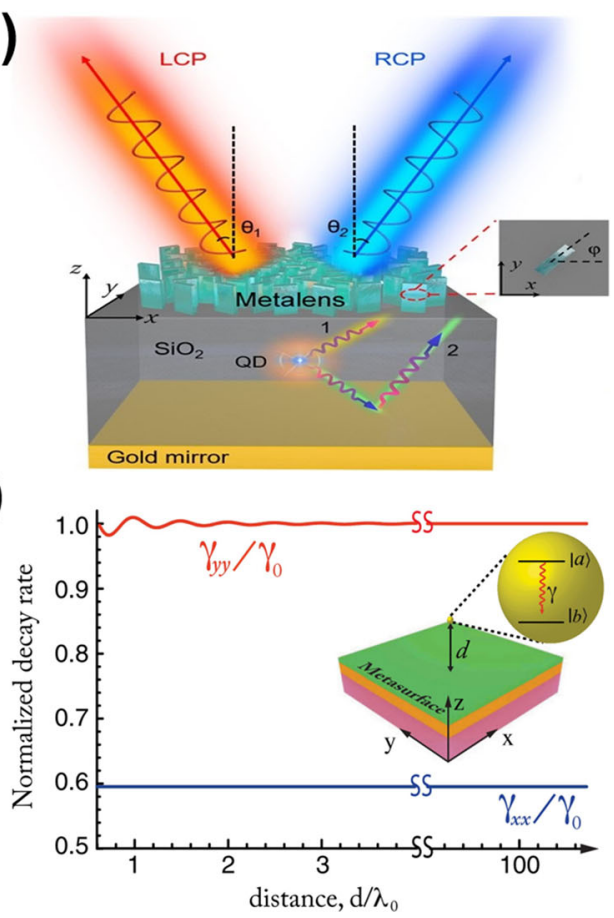

Fig. 9 The basic concepts and Purcell enhancement of metasurface. a The SEM image of typical metasurface [301]. The schematic diagrams of controlling the (b) phases [302], (c) frequencies [303], and (d) polarizations [304] of electric field by metasurface. e Metasurface controlling the light polarization for anisotropic quantum vacuum [305]. f Anisotropic Purcell factors of $x$ - and $y$-polarized emitters in the metasurface (e) [305]

through controlling the polarized light reflected by metasurface [Fig. 9(e)]. Thus the local densities of optical states in $x$ - and $y$-directions are different, i.e., anisotropic Purcell enhancement occurs [Fig. 9(f)], and Purcell factors of $y$-polarized emitters are larger than those of $x$-polarized emitters. This anisotropic Purcell enhancement can be applied to obtain valley coherence [310], long-lifetime coherence [311], and coherent manipulation [312]. Also, the specific polarization of single photon emission can be generated by other designs of metasurfaces, like bullseye structures metasurface with radial phase distribution [324], and metasurface-based spin-splitting bifocal lens [304].

\section{Spontaneous emission in epsilon-near-zero materials}

Epsilon-near-zero (ENZ) material refers to the material with a dielectric constant $\epsilon=0$. The materials with zero-dielectric constant have been experimentally realized in various 
wavelengths [325-329], such as doped semiconductors in the near-infrared band, topological insulators in the ultraviolet, and polarized materials in the mid-infrared [327, 330332]. If the propagation constant of the waveguide working at the cutoff frequency is zero, its effective propagation wavelength will be infinite, which is equivalent to an ENZ material [333].

Because of zero permittivity, ENZ materials possess extraordinary electromagnetic properties, such as electromagnetic tunneling [292, 334-336], directional radiation [337, 338], nonlinear effect [339-343], boundary effect [344-346], and resonance stacking effect [330, 347]. One special ability of ENZ materials is suppressing vacuum fluctuation, so the cavity embedded in ENZ materials can be used to control light-matter interaction [133, 348]. An optical cavity embedded within open ENZ materials has geometrically invariant eigenmodes, which is beneficial to the generation of deformable resonant devices [349].

In most photonic structures, Purcell factor is very sensitive to the position of the quantum emitter. It always needs an accurate positioning, which is difficult to be realized in the experiments. While in ENZ materials, Purcell factor is not much relative to the emitter's position because of a uniform local density of states [350]. Focused local field in ENZ plates is conductive to the emission of quantum emitter [351,352]. SE of emitters in ENZ materials can be greatly improved by Dicke superradiation effect [353]. The air cavity embedded in the open ENZ materials has two modes of radiation and non-radiation [Fig. 10(a)], which can enhance or suppress the SE of the emitter in the cavity [Fig. 10(b)] [354]. For the application to the single-photon source, the directional radiation can be realized within the zero-index metamaterial [Figs. 10(c, d)] [337, 338, 355].

(a)
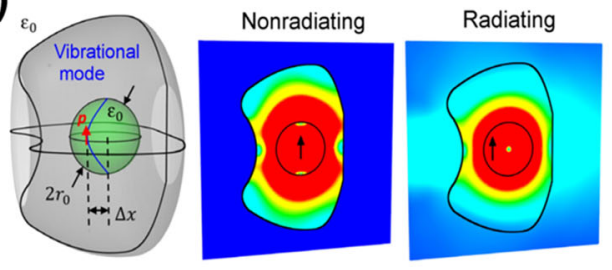

(c)

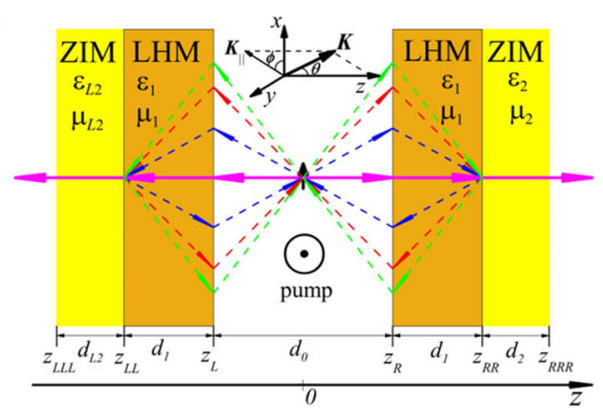

(b)

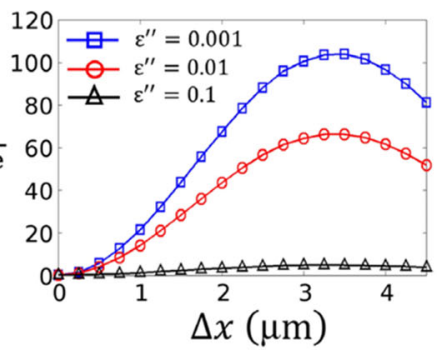

(d)

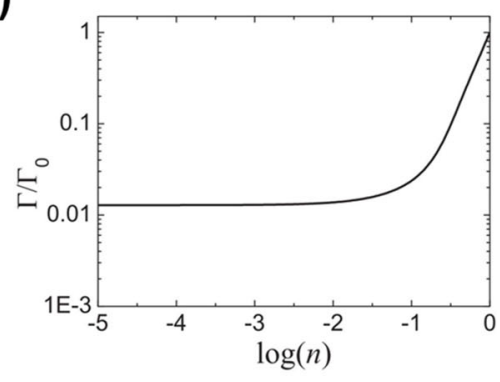

Fig. 10 a Sketch of air cavity embedded in the open ENZ materials with nonradiating and radiating modes [354]. b Purcell factors for different amounts of loss $\left(\varepsilon^{\prime \prime}\right)$ in the ENZ medium in structure (a) [354]. c Sketch of optical cavity made of zero-index metamaterial [337]. d Purcell factor of directional single photon source in structure (c) [337] 


\section{Spontaneous emission in hybrid structures}

In the study of Purcell effect, the assembly of hybrid structures from different basic photonic components can achieve many advances superior to those of the individual photonic subunits [5]. In the sections above, we mainly focused on the properties of spontaneous emission in micro- or nanostructures only with single photonic component. However, in the practical CQED applications, hybrid structures composed of multi-photonic-entities are required, such as gap plasmon structures combining WGMs [249] or metamaterials [250]. So far, based on various photonic components, Purcell enhancement can be obtained with novel characteristics like chirality [148] and topological protection [238].

Hybrid structures made up of PCs and plasmonic devices always attract people's attention. In a hybrid photonic structure that consists of a gold dimer placed on the top of a 1D PC cavity, Purcell factors of $0 \sim 4.9 \times 10^{3}$ are available [356], which can be applied as a wonderful switching knob. However, it's a challenge to couple photons from a circularly polarized emitter into photonic structure to simultaneously realize strong spontaneous emission enhancement and unidirectional propagation locked by helicity of optical mode. By combining a photonic crystal and metallic nanoparticle structure [Fig. 11(a)] to create nanocavities with both strong local-field intensity and high helicity [Fig. 11(b)] [148], the Purcell factor of circularly polarized photons emitting into the photonic crystal waveguide reaches 148 [Fig. 11(c)], which is one order of magnitude larger than that without the nanoparticle, and with $\approx 95 \%$ of photons propagating unidirectionally along the waveguide [Fig. 11(c)]. This nanophotonic interface of chiral quantum electrodynamics can be applied in on-chip nonreciprocal quantum light sources, quantum circuits, and scalable quantum networks.

Topological states are some unique optical modes between the optical bands, described by topological invariants in the reciprocal space [357-359]. Recently, topological protection has been introduced into the Purcell enhancement with the discovery of mechanism of edge state-led mode coupling under topological protection [238], that localized surface plasmons almost do not have any influence on the edge state, while the edge state greatly changes the local field distribution of surface plasmons [Fig. 11(e)]. Based on this mechanism, in the well-designed topological photonic structure containing a resonant plasmon nanoantenna [Fig. 11(d)], an obvious absorption reduction in the spontaneous emission spectra appears due to the near-field deformation around the antenna induced by the edge state [Figs. 11(f, g)]. Because a plasmon antenna with ultrasmall mode volume provides large Purcell enhancement and simultaneously the photonic crystal guides almost all scattering light into its edge state, the rate of nonscattering single photons reaches more than $10^{4} \gamma_{0}$ [Fig. $\left.11(\mathrm{~g})\right]$. Nonscattering large Purcell enhancement will provide practical use for on-chip quantum light sources, such as single-photon sources and nanolasers.

\section{Summary and prospects}

We have reviewed the spontaneous emission enhancement based on the CQED principle in micro- or nanophotonic structures, including whisper gallery microcavities, photonic crystals, surface plasmon metallic nanostructures, metamaterials, and hybrid structures. By carefully designed optical modes in micro- or nanostructures, spontaneous emission enhancement can be obtained with different peculiarities. Whispering gallery microcavities provide high $Q$, thus large Purcell factors can be obtained easily. PCs require defects and waveguides to create a cavity, which can improve photon collection but 
(a)

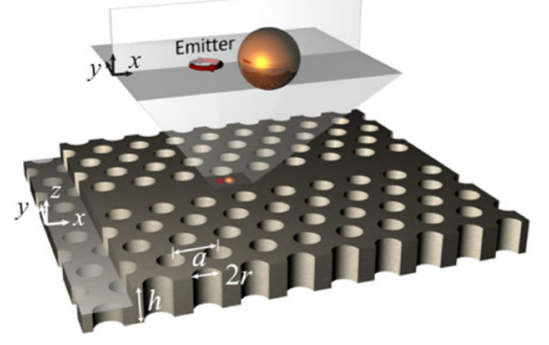

(c)

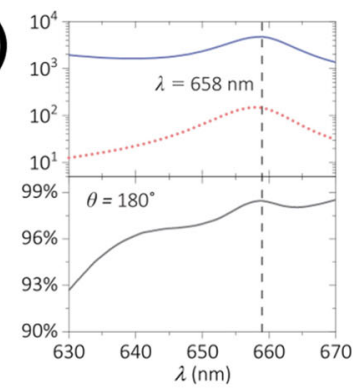

(b)
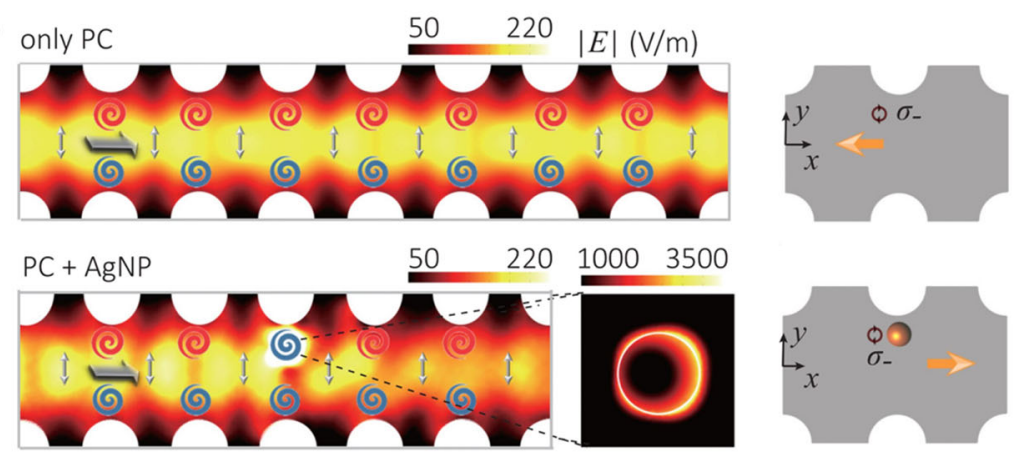

(d)

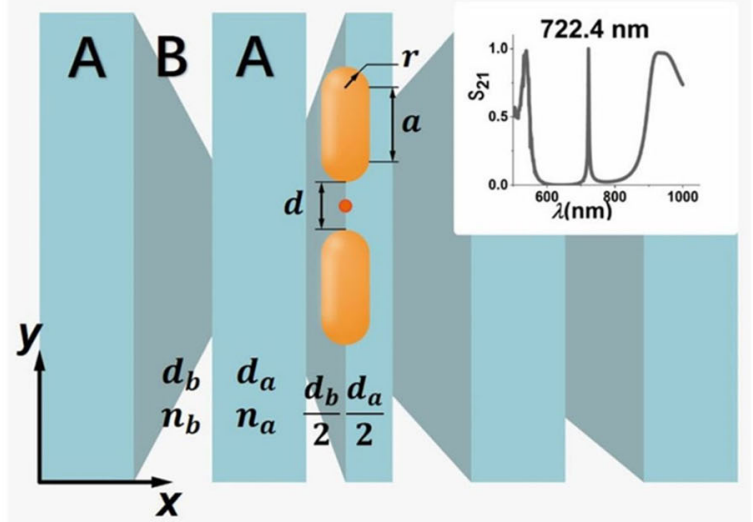

(e)

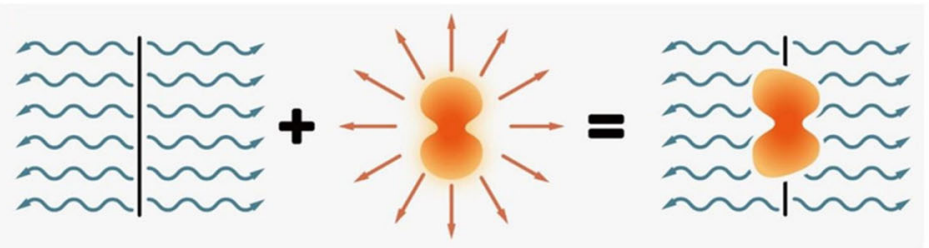

(f)
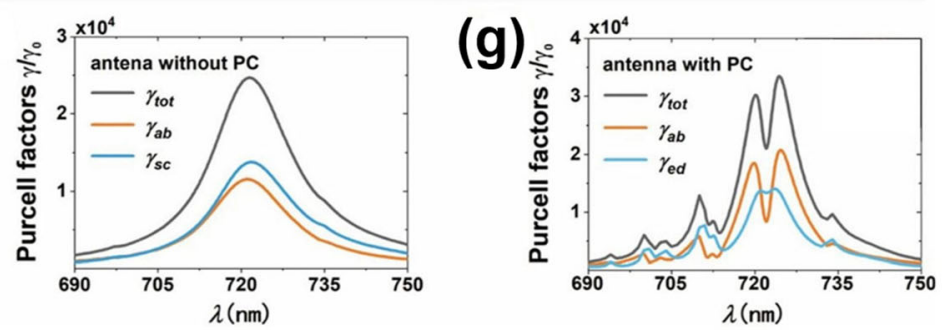

Fig. 11 Purcell effect in hybrid structures. a Schematic diagram of coupled structure consisting of PC and silver nanoparticle coupled to a chiral polarized emitter [148]. b Mechanism of spin-locked photonic propagation with chiral emitter [148]. c Purcell factors of structure (a) with high directionality [148]. d Schematic diagram of 1D topological structure including a silver nanoantenna and a quantum emitter [238]. e Mechanism of edge state-led mode coupling [238]. Purcell factors of the nanoantenna (f) without and (g) with topological structure [238] 
Purcell enhancement is not very large. By using novel PCs like Dirac/Weyl-type lattices, people may find Purcell enhancement with more interesting characteristics. Surface plasmon nanostructures offer large Purcell factors in the nanoscale confined space, but the metallic loss is always a barrier. As for metamaterials, metasurfaces focus on controlling the phase and polarization of emitted photons, while ENZ materials keep the emission enhancement away from influence of emitters' positions. But there are still lots of research gaps that should be filled for Purcell effect in metamaterials. Hybrid photonic structures allow some unique characteristics related to spontaneous emission enhancement superior to single photonic entity. It's worth looking forward to more unpredictable innovations on spontaneous emission enhancement in more hybrid photonic structures. Overall, achievements of spontaneous emission enhancement in micro- or nanophotonic structures can be utilized in the on-chip quantum information process, quantum states controlling, and quantum internet.

It's worth stressing that interdisciplinary between quantum science and micro- or nanophotonics has become the engine of innovation with the state-of-art nanotechnology. Single-photon source based on Purcell enhancement is the basic building block of on-chip quantum information, which can be applied in quantum gates [360], quantum nodes [4], nanolasers [361], and so on. Also, brand-new characteristics from hybrid structures are introduced to single photons especially topological protection $[238,362-$ 365]. By designing open symmetric cavities with both gain and loss mechanisms [366], one could find amazing additional nature of single photons enhancement at exceptional points in PT-symmetry systems. Besides CQED, more and more quantum areas have been combined with micro- or nanophotonics. For quantum beam splitters [367], if the enhancement of photons exists, it may be easier to observe the Hong-Ou-Mandel interferometry effect, which will provide new way to engineer the quantum states. For quantum holography [368], enhanced entangled photons may lead to the better resolution and less noise. For quantum key distribution [369], it may help lower symbol error rate so that the efficiency of quantum information transport can be improved. Therefore, the subject integration between quantum science and micro- or nanophotonics will be an unstoppable trend.

\section{Abbreviations}

CQED: Cavity quantum electrodynamics; SE: Spontaneous emission; SPP: Surface plasmon polariton; WGM: Whisper gallery mode; PC: Photonic crystal; PBG: Photonic band gap; SEM: Scanning electron microscope; ENZ: epsilon-near-zero; $\mathrm{NV}$ : nitrogen-vacancy

\section{Acknowledgements}

Not applicable.

\section{Authors' contributions}

Ying Gu provided a guided writing. Zhiyuan Qian wrote the entire manuscript and prepared the figures. Lingxiao Shan joined the part of photonic crystals. Xinchen Zhang joined the part of whisper gallery modes. Yun Ma and Qi Liu joined the part of metamaterials. All authors read and approved the final manuscript.

Funding

This work is supported by the National Natural Science Foundation of China under Grants No. 11974032, No. 11525414, and No. 11734001, and by the Key R\&D Program of Guangdong Province under Grant No. 2018 B030329001.

Availability of data and materials

Data sharing is not applicable to this article as no new data were created or analysed in this study.

\section{Declarations}




\section{Author details}

${ }^{1}$ State Key Laboratory for Mesoscopic Physics, Department of Physics, Peking University, Beijing, China. ${ }^{2}$ Frontiers Science Center for Nano-optoelectronics, Collaborative Innovation Center of Quantum Matter, and Beijing Academy of Quantum Information Sciences, Peking University, Beijing, China. ${ }^{3}$ Collaborative Innovation Center of Extreme Optics, Shanxi University, Taiyuan, China. ${ }^{4}$ Beijing Academy of Quantum Information Sciences, Peking University, Beijing, China. ${ }^{5}$ Peking University Yangtze Delta Institute of Optoelectronics, Nantong, China.

Received: 28 June 2021 Accepted: 3 August 2021

Published online: 16 September 2021

\section{References}

1. Purcell E. Spontaneous emission probabilities at radio frequencies. Phys Rev. 1946;69:681.

2. Haroche S, Kleppner D. Cavity quantum electrodynamics. Phys Today. 1989;42(1):24-30.

3. Vahala KJ. Optical microcavities. Nature. 2003;424:839-46.

4. Kimble HJ. The quantum internet. Nature. 2008;453:1023-30.

5. Benson O. Assembly of hybrid photonic architectures from nanophotonic constituents. Nature. 2011;480:193-9.

6. Jacob Z, Shalaev VM. Plasmonics goes quantum. Science. 2011;334(6055):463-4. https://doi.org/10.1126/science. 1211736. https://science.sciencemag.org/content/334/6055/463.full.pdf.

7. Tame MS, McEnery K, Özdemir Ş, Lee J, Maier SA, Kim M. Quantum plasmonics. Nat Phys. 2013;9(6):329-40.

8. Sauvan C, Hugonin JP, Maksymov IS, Lalanne P. Theory of the spontaneous optical emission of nanosize photonic and plasmon resonators. Phys Rev Lett. 2013;110:237401. https://doi.org/10.1103/PhysRevLett.110.237401.

9. Zhu J, Ozdemir SK, Xiao Y-F, Li L, He L, Chen D-R, Yang L. On-chip single nanoparticle detection and sizing by mode splitting in an ultrahigh-q microresonator. Nat Photonics. 2010;4(1):46.

10. Kiraz A, Michler P, Becher C, Gayral B, Imamoǧlu A, Zhang L, Hu E, Schoenfeld WV, Petroff PM. Cavity-quantum electrodynamics using a single inas quantum dot in a microdisk structure. Appl Phys Lett. 2001;78(25):3932-4. https://doi.org/10.1063/1.1379987.

11. Kippenberg TJ, Spillane SM, Vahala KJ. Demonstration of ultra-high-q small mode volume toroid microcavities on a chip. Appl Phys Lett. 2004;85(25):6113-5. https://doi.org/10.1063/1.1833556.

12. Akahane Y, Asano T, Song B-S, Noda S. High-Q photonic nanocavity in a two-dimensional photonic crystal. Nature. 2003:425(6961):944-7. https://doi.org/10.1038/nature02063. Accessed 12 Jan 2021.

13. Asano T, Song B-S, Noda S. Analysis of the experimental Q factors ( 1 million) of photonic crystal nanocavities. Opt Express. 2006;14(5):1996. https://doi.org/10.1364/OE.14.001996. Accessed 22 Feb 2021.

14. Lakhani AM, Kim M-K, Lau EK, Wu MC. Plasmonic crystal defect nanolaser. Opt Express. 2011;19(19):18237. https:// doi.org/10.1364/OE.19.018237. Accessed 20 March 2021.

15. Altug H, Englund D, Vučković J. Ultrafast photonic crystal nanocavity laser. Nat Phys. 2006;2(7):484-8. https://doi. org/10.1038/nphys343. Accessed 11 Jan 2021.

16. Kinkhabwala A, Yu Z, Fan S, Avlasevich Y, Müllen K, Moerner W. Large single-molecule fluorescence enhancements produced by a bowtie nanoantenna. Nat Photonics. 2009;3(11):654-7.

17. Barnes WL, Dereux A, Ebbesen TW. Surface plasmon subwavelength optics. Nature. 2003;424:824-30.

18. Maier SA. Plasmonics: Fundamentals and Applications. New York: Springer; 2007.

19. Chikkaraddy R, De Nijs B, Benz F, Barrow SJ, Scherman OA, Rosta E, Demetriadou A, Fox P, Hess O, Baumberg JJ. Single-molecule strong coupling at room temperature in plasmonic nanocavities. Nature. 2016;535(7610):127-30.

20. Lian H, GuY, Ren J, Zhang F, Wang L, Gong Q. Efficient single photon emission and collection based on excitation of gap surface plasmons. Phys Rev Lett. 2015;114:193002. https://doi.org/10.1103/PhysRevLett.114.193002.

21. Jaynes ET, Cummings FW. Comparison of quantum and semiclassical radiation theories with application to the beam maser. Proc IEEE. 1963;51(1):89-109. https://doi.org/10.1109/PROC.1963.1664.

22. Guerlin C, Brion E, Esslinger T, Mølmer K. Cavity quantum electrodynamics with a rydberg-blocked atomic ensemble. Phys Rev A. 2010;82:053832. https://doi.org/10.1103/PhysRevA.82.053832.

23. Sanchez-Mondragon JJ, Narozhny NB, Eberly JH. Theory of spontaneous-emission line shape in an ideal cavity. Phys Rev Lett. 1983;51:550-3. https://doi.org/10.1103/PhysRevLett.51.550.

24. Hulet RG, Hilfer ES, Kleppner D. Inhibited spontaneous emission by a rydberg atom. Phys Rev Lett. 1985;55: 2137-40. https://doi.org/10.1103/PhysRevLett.55.2137.

25. Yokoyama H. Physics and device applications of optical microcavities. Science. 1992;256(5053):66-70. https://doi. org/10.1126/science.256.5053.66.

26. Kimble HJ. Strong interactions of single atoms and photons in cavity QED. Phys Scr. 1998;T76(1):127. https://doi. org/10.1238/physica.topical.076a00127.

27. Nußmann S, Hijlkema M, Weber B, Rohde F, Rempe G, Kuhn A. Submicron positioning of single atoms in a microcavity. Phys Rev Lett. 2005;95:173602. https://doi.org/10.1103/PhysRevLett.95.173602.

28. Bourdel T, Donner T, Ritter S, Öttl A, Köhl M, Esslinger T. Cavity qed detection of interfering matter waves. Phys Rev A. 2006;73:043602. https://doi.org/10.1103/PhysRevA.73.043602.

29. Wilk T, Webster SC, Specht HP, Rempe G, Kuhn A. Polarization-controlled single photons. Phys Rev Lett. 2007;98: 063601. https://doi.org/10.1103/PhysRevLett.98.063601.

30. Brennecke F, Donner T, Ritter S, Bourdel T, Köhl M, Esslinger T. Cavity qed with a bose-einstein condensate. Nature. 2007:450(7167):268-71.

31. Brune M, Hagley E, Dreyer J, Maître X, Maali A, Wunderlich C, Raimond JM, Haroche S. Observing the progressive decoherence of the "meter" in a quantum measurement. Phys Rev Lett. 1996;77:4887-90. https://doi.org/10.1103/ PhysRevLett.77.4887.

32. Haroche S. Nobel lecture: Controlling photons in a box and exploring the quantum to classical boundary. Rev Mod Phys. 2013;85:1083-102. https://doi.org/10.1103/RevModPhys.85.1083.

33. Lounis B, Orrit M. Single-photon sources. Rep Prog Phys. 2005;68(5):1129-79. https://doi.org/10.1088/0034-4885/ $68 / 5 /$ r04. 
34. Miller R, Northup TE, Birnbaum KM, Boca A, Boozer AD, Kimble HJ. Trapped atoms in cavity QED: coupling quantized light and matter. J Phys B Atomic Mol Phys. 2005;38(9):551-65. https://doi.org/10.1088/0953-4075/38/9/ 007.

35. Walther H, Varcoe BTH, Englert B-G, Becker T. Cavity quantum electrodynamics. Rep Prog Phys. 2006;69(5): 1325-82. https://doi.org/10.1088/0034-4885/69/5/r02.

36. Reiserer A, Rempe G. Cavity-based quantum networks with single atoms and optical photons. Rev Mod Phys. 2015;87:1379-418. https://doi.org/10.1103/RevModPhys.87.1379.

37. Walther H. Experiments on cavity quantum electrodynamics. Phys Rep. 1992;219(3-6):263-81.

38. Berman PR, (ed). Cavity Quantum Electrodynamics. New York: Academic Press; 1993.

39. Mabuchi H, Doherty A. Cavity quantum electrodynamics: coherence in context. Science. 2002;298(5597):1372-7.

40. Dowling JP. Exploring the quantum: Atoms, cavities, and photons. Am J Phys. 2014;82(1):86-7. https://doi.org/10. $1119 / 1.4827830$.

41. Miller R, Northup TE, Birnbaum KM, Boca A, Boozer AD, Kimble HJ. Trapped atoms in cavity QED: coupling quantized light and matter. J Phys B Atomic Mol Phys. 2005;38(9):551-65. https://doi.org/10.1088/0953-4075/38/9/ 007.

42. Casten R. Shape phase transitions and critical-point phenomena in atomic nuclei. Nat Phys. 2006;2(12):811-20.

43. Carmichael HJ. Statistical Methods in Quantum Optics. Berlin: Springer; 2008.

44. Meystre P, SargentIII M. Elements of Quantum Optics. Berlin Heidelberg: Springer; 1990.

45. Ren J, Gu Y, Zhao D, Zhang F, Zhang T, Gong Q. Evanescent-vacuum-enhanced photon-exciton coupling and fluorescence collection. Phys Rev Lett. 2017;1 18:073604. https://doi.org/10.1103/PhysRevLett.1 18.073604.

46. Svelto O, Hanna DC. Principles of Lasers. New York: Springer; 2010

47. Scully MO, Zubairy MS, et al. Quantum Optics. Cambridge, UK: Cambridge University Press; 1997.

48. Cohen-Tannoudji C, Reynaud S. Dressed-atom description of resonance fluorescence and absorption spectra of a multi-level atom in an intense laser beam. J Phys B Atomic Mol Phys. 1977;10(3):345-63. https://doi.org/10.1088/ 0022-3700/10/3/005.

49. Zhang S-S, Cheng H, Xin P-P, Wang H-M, Xu Z-S, Liu H-P. A sensitive detection of high rydberg atom with large dipole moment. Chin Phys B. 2018;27(7):074207. https://doi.org/10.1088/1674-1056/27/7/074207.

50. Saffman M, Walker TG, Mølmer K. Quantum information with rydberg atoms. Rev Mod Phys. 2010;82:2313-63. https://doi.org/10.1103/RevModPhys.82.2313.

51. Ridolfo A, Di Stefano O, Fina N, Saija R, Savasta S. Quantum plasmonics with quantum dot-metal nanoparticle molecules: Influence of the fano effect on photon statistics. Phys Rev Lett. 2010;105:263601. https://doi.org/10. 1103/PhysRevLett.105.263601.

52. Yalla R, Le Kien F, Morinaga M, Hakuta K. Efficient channeling of fluorescence photons from single quantum dots into guided modes of optical nanofiber. Phys Rev Lett. 2012;109:063602. https://doi.org/10.1103/PhysRevLett.109. 063602.

53. Birowosuto MD, Yokoo A, Zhang G, Tateno K, Kuramochi E, Taniyama H, Takiguchi M, Notomi M. Movable high-q nanoresonators realized by semiconductor nanowires on a si photonic crystal platform. Nat Mater. 2014;13(3):279-85.

54. Aharonovich I, Englund D, Toth M. Solid-state single-photon emitters. Nat Photonics. 2016;10(10):631-41.

55. Leistikow MD, Mosk AP, Yeganegi E, Huisman SR, Lagendijk A, Vos WL. Inhibited spontaneous emission of quantum dots observed in a 3d photonic band gap. Phys Rev Lett. 2011;107:193903. https://doi.org/10.1103/ PhysRevLett.107.193903.

56. Claudon J, Bleuse J, Malik NS, Bazin M, Jaffrennou P, Gregersen N, Sauvan C, Lalanne P, Gérard J-M. A highly efficient single-photon source based on a quantum dot in a photonic nanowire. Nat Photonics. 2010;4(3):174-7.

57. Bleuse J, Claudon J, Creasey M, Malik NS, Gérard J-M, Maksymov I, Hugonin J-P, Lalanne P. Inhibition, enhancement, and control of spontaneous emission in photonic nanowires. Phys Rev Lett. 2011;106:103601. https://doi.org/10.1103/PhysRevLett.106.103601.

58. Liu X, Asano T, Odashima S, Nakajima H, Kumano H, Suemune I. Bright single-photon source based on an inas quantum dot in a silver-embedded nanocone structure. Appl Phys Lett. 2013;102(13):131114. https://doi.org/10. 1063/1.4801334.

59. Liu J, Su R, Wei Y, Yao B, da Silva SFC, Yu Y, Iles-Smith J, Srinivasan K, Rastelli A, Li J, et al. A solid-state source of strongly entangled photon pairs with high brightness and indistinguishability. Nat Nanotechnol. 2019;14(6):586-93.

60. Kuhn A, Hennrich M, Rempe G. Deterministic single-photon source for distributed quantum networking. Phys Rev Lett. 2002;89:067901. https://doi.org/10.1103/PhysRevLett.89.067901.

61. Boozer AD, Boca A, Miller R, Northup TE, Kimble HJ. Reversible state transfer between light and a single trapped atom. Phys Rev Lett. 2007:98:193601. https://doi.org/10.1103/PhysRevLett.98.193601.

62. Klimov W, Ducloy M. Spontaneous emission rate of an excited atom placed near a nanofiber. Phys Rev A. 2004;69: 013812. https://doi.org/10.1103/PhysRevA.69.013812.

63. Yalla R, Sadgrove M, Nayak KP, Hakuta K. Cavity quantum electrodynamics on a nanofiber using a composite photonic crystal cavity. Phys Rev Lett. 2014;113:143601. https://doi.org/10.1103/PhysRevLett.113.143601.

64. Maksymov IS, Besbes M, Hugonin JP, Yang J, Beveratos A, Sagnes I, Robert-Philip I, Lalanne P. Metal-coated nanocylinder cavity for broadband nonclassical light emission. Phys Rev Lett. 2010;105:180502. https://doi.org/10. 1103/PhysRevLett.105.180502.

65. Suemune I, Nakajima H, Liu X, Odashima S, Asano T, lijima H, Huh J-H, Idutsu Y, Sasakura H, Kumano H. Metal-coated semiconductor nanostructures and simulation of photon extraction and coupling to optical fibers for a solid-state single-photon source. Nanotechnology. 2013;24(45):455205. https://doi.org/10.1088/0957-4484/24/ 45/455205.

66. Merkel B, Ulanowski A, Reiserer A. Coherent and purcell-enhanced emission from erbium dopants in a cryogenic high-q resonator. Phys Rev X. 2020;10:041025. https://doi.org/10.1103/PhysRevX.10.041025.

67. Goban A, Hung C-L, Hood JD, Yu S-P, Muniz JA, Painter O, Kimble HJ. Superradiance for atoms trapped along a photonic crystal waveguide. Phys Rev Lett. 2015;115:063601. https://doi.org/10.1103/PhysRevLett.115.063601. 
68. Mitsch R, Sayrin C, Albrecht B, Schneeweiss P, Rauschenbeutel A. Quantum state-controlled directional spontaneous emission of photons into a nanophotonic waveguide. Nat Commun. 2014;5(1):1-5.

69. Yang S, Wang Y, Sun H. Advances and prospects for whispering gallery mode microcavities. Adv Opt Mater. 2015;3(9):1136-62. https://doi.org/10.1002/adom.201500232.

70. Zou C, Dong C, Cui J, Sun F, Yang Y, Wu X, Han Z, Guo G. Whispering gallery mode optical microresonators: fundamentals and applications. Sci Sinica Phys Mech Astron. 2012;42(11):1155-75.

71. Matsko AB, Ilchenko VS. Optical resonators with whispering-gallery modes-part i: basics. IEEE J Sel Top Quant Electron. 2006;12(1):3-14. https://doi.org/10.1109/JSTQE.2005.862952.

72. Ilchenko VS, Matsko AB. Optical resonators with whispering-gallery modes-part ii: applications. IEEE J Sel Top Quant Electron. 2006;12(1):15-32. https://doi.org/10.1109/JSTQE.2005.862943.

73. Rayleigh L. CXII. the problem of the whispering gallery. Lond Edinb Dublin Philos Mag J Sci. 1910;20(120):1001-4. https://doi.org/10.1080/14786441008636993.

74. Richtmyer RD. Dielectric resonators. J Appl Phys. 1939;10(6):391-8. https://doi.org/10.1063/1.1707320.

75. Ashkin A, Dziedzic JM. Observation of resonances in the radiation pressure on dielectric spheres. Phys Rev Lett. 1977;38:1351-4. https://doi.org/10.1103/PhysRevLett.38.1351.

76. Benner RE, Barber PW, Owen JF, Chang RK. Observation of structure resonances in the fluorescence spectra from microspheres. Phys Rev Lett. 1980;44:475-8. https://doi.org/10.1103/PhysRevLett.44.475.

77. Garrett CGB, Kaiser W, Bond WL. Stimulated emission into optical whispering modes of spheres. Phys Rev. 1961;124:1807-9. https://doi.org/10.1103/PhysRev.124.1807.

78. McCall SL, Levi AFJ, Slusher RE, Pearton SJ, Logan RA. Whispering-gallery mode microdisk lasers. Appl Phys Lett. 1992;60(3):289-91. https://doi.org/10.1063/1.106688.

79. Gorodetsky ML, Savchenkov AA, Ilchenko VS. Ultimate q of optical microsphere resonators. Opt Lett. 1996;21(7): 453-5. https://doi.org/10.1364/OL.21.000453.

80. Lefèvre-Seguin V, Haroche S. Towards cavity-qed experiments with silica microspheres. Mater Sci Eng B. 1997;48(1):53-8. https://doi.org/10.1016/S0921-5107(97)00080-9.

81. Vernooy DW, Ilchenko VS, Mabuchi H, Streed EW, Kimble HJ. High-q measurements of fused-silica microspheres in the near infrared. Opt Lett. 1998;23(4):247-9. https://doi.org/10.1364/OL.23.000247.

82. Vernooy DW, Furusawa A, Georgiades NP, Ilchenko VS, Kimble HJ. Cavity qed with high-q whispering gallery modes. Phys Rev A. 1998;57:2293-6. https://doi.org/10.1103/PhysRevA.57.R2293.

83. Chen W, Özdemir ŞK, Zhao G, Wiersig J, Yang L. Exceptional points enhance sensing in an optical microcavity. Nature. 2017:548(7666):192-6.

84. Michler P. A Quantum Dot Sing le-Photon Turnstile Device. Science. 2000;290(5500):2282-5. https://doi.org/10. 1126/science.290.5500.2282. Accessed 19 Oct 2020.

85. Moreau E, Robert I, Gérard J, Abram I, Manin L, Thierry-Mieg V. Single-mode solid-state single photon source based on isolated quantum dots in pillar microcavities. Appl Phys Lett. 2001;79(18):2865-7.

86. Santori C, Fattal D, Vučković J, Solomon GS, Yamamoto Y. Indistinguishable photons from a single-photon device. Nature. 2002;419(6907):594-7.

87. Pelton M, Santori C, Vucković J, Zhang B, Solomon GS, Plant J, Yamamoto Y. Efficient source of single photons: A single quantum dot in a micropost microcavity. Phys Rev Lett. 2002;89:233602. https://doi.org/10.1103/ PhysRevLett.89.233602.

88. Faraon A, Barclay PE, Santori C, Fu K-MC, Beausoleil RG. Resonant enhancement of the zero-phonon emission from a colour centre in a diamond cavity. Nat Photonics. 2011;5(5):301.

89. Artemyev MV, Woggon U, Wannemacher R, Jaschinski H, Langbein W. Light trapped in a photonic dot: Microspheres act as a cavity for quantum dot emission. Nano Lett. 2001;1(6):309-14. https://doi.org/10.1021/ nl015545l.

90. Bayer M, Reinecke TL, Weidner F, Larionov A, McDonald A, Forchel A. Inhibition and enhancement of the spontaneous emission of quantum dots in structured microresonators. Phys Rev Lett. 2001;86:3168-71. https://doi. org/10.1103/PhysRevLett.86.3168.

91. Armani D, Kippenberg T, Spillane S, Vahala K. Ultra-high-q toroid microcavity on a chip. Nature. 2003;421(6926): 925-8.

92. Spillane SM, Kippenberg TJ, Painter OJ, Vahala KJ. Ideality in a fiber-taper-coupled microresonator system for application to cavity quantum electrodynamics. Phys Rev Lett. 2003;91:043902. https://doi.org/10.1103/ PhysRevLett.91.043902.

93. Louyer $Y$, Meschede D, Rauschenbeutel A. Tunable whispering-gallery-mode resonators for cavity quantum electrodynamics. Phys Rev A. 2005;72:031801. https://doi.org/10.1103/PhysRevA.72.031801.

94. Reithmaier JP, Sek G, Löffler A, Hofmann C, Kuhn S, Reitzenstein S, Keldysh L, Kulakovskii V, Reinecke T, Forchel A. Strong coupling in a single quantum dot-semiconductor microcavity system. Nature. 2004;432(7014):197-200.

95. Peter E, Senellart P, Martrou D, Lemaître A, Hours J, Gérard JM, Bloch J. Exciton-photon strong-coupling regime for a single quantum dot embedded in a microcavity. Phys Rev Lett. 2005;95:067401. https://doi.org/10.1103/ PhysRevLett.95.067401.

96. Park Y-S, Cook AK, Wang H. Cavity qed with diamond nanocrystals and silica microspheres. Nano Lett. 2006;6(9): 2075-9. https://doi.org/10.1021/nl061342r. PMID: 16968028.

97. Aoki T, Dayan B, Wilcut E, Bowen WP, Parkins AS, Kippenberg T, Vahala K, Kimble H. Observation of strong coupling between one atom and a monolithic microresonator. Nature. 2006;443(7112):671-4.

98. Eisaman MD, Fan J, Migdall A, Polyakov SV. Invited review article: Single-photon sources and detectors. Rev Sci Instrum. 2011;82(7):071101. https://doi.org/10.1063/1.3610677.

99. Shen J-T, Fan S. Theory of single-photon transport in a single-mode waveguide. ii. coupling to a whispering-gallery resonator containing a two-level atom. Phys Rev A. 2009;79:023838. https://doi.org/10.1103/PhysRevA.79.023838.

100. Hartmann MJ, Brandao FG, Plenio MB. Strongly interacting polaritons in coupled arrays of cavities. Nat Phys. 2006;2(12):849-55. 
101. Xiao Y-F, Lin X-M, Gao J, Yang Y, Han Z-F, Guo G-C. Realizing quantum controlled phase flip through cavity qed. Phys Rev A. 2004;70:042314. https://doi.org/10.1103/PhysRevA.70.042314.

102. Joannopoulos JD, Johnson SG, Winn JN, Meade RD. Photonic Crystals-molding the Flow of Light, 2nd edn. New Jersey: Princeton University Press; 2008.

103. David A, Benisty H, Weisbuch C. Photonic crystal light-emitting sources. Rep Prog Phys. 2012;75(12):126501. https://doi.org/10.1088/0034-4885/75/12/126501. Accessed 14 Jan 2021.

104. Notomi M. Manipulating light with strongly modulated photonic crystals. Rep Prog Phys. 2010;73(9):096501. https://doi.org/10.1088/0034-4885/73/9/096501. Accessed 14 Jan 2021.

105. Song B-S, Noda S, Asano T, Akahane Y. Ultra-high-Q photonic double-heterostructure nanocavity. Nat Mater. 2005;4(3):207-10. https://doi.org/10.1038/nmat1320. Accessed 12 Jan 2021.

106. Yablonovitch E. Inhibited Spontaneous Emission in Solid-State Physics and Electronics. Phys Rev Lett. 1987;58(20): 2059-62. https://doi.org/10.1103/PhysRevLett.58.2059. Accessed 11 Jan 2021.

107. Krauss T. Photonic crystals in the optical regime - past, present and future. Prog Quantum Electron. 1999;23(2): 51-96. https://doi.org/10.1016/S0079-6727(99)00004-X. Accessed 11 Jan 2021.

108. Song D-S, Kim S-H, Park H-G, Kim C-K, Lee Y-H. Single-fundamental-mode photonic-crystal vertical-cavity surface-emitting lasers. Appl Phys Lett. 2002;80(21):3901-3. https://doi.org/10.1063/1.1481984. Accessed 11 Jan 2021.

109. Yablonovitch E, Gmitter TJ, Meade RD, Rappe AM, Brommer KD, Joannopoulos JD. Donor and acceptor modes in photonic band structure. Phys Rev Lett. 1991;67(24):3380-3. https://doi.org/10.1103/PhysRevLett.67. 3380. Accessed 11 Jan 2021.

110. Painter O. Two-Dimensional Photonic Band-Gap Defect Mode Laser. Science. 1999;284(5421):1819-21. https://doi. org/10.1126/science.284.5421.1819. Accessed 11 Jan 2021.

111. Ogawa S, Imada M, Yoshimoto S, Okano M, Noda S. Control of Light Emission by 3D Photonic Crystals. Science. 2004:305(5681):227-9. https://doi.org/10.1126/science.1097968. Accessed 10 March 2021.

112. Lončar M, Yoshie T, Scherer A, Gogna P, Qiu Y. Low-threshold photonic crystal laser. Appl Phys Lett. 2002;81 (15): 2680-2. https://doi.org/10.1063/1.1511538. Accessed 11 Jan 2021.

113. Zhou W, Sabarinathan J, Bhattacharya P, Kochman B, Berg EW, Yu P-C, Pang SW. Characteristics of a photonic bandgap single defect microcavity electroluminescent device. IEEE J Quantum Electron. 2001;37(9):1153-60. https://doi.org/10.1109/3.945320. Accessed 11 Jan 2021.

114. Park H-G, Hwang J-K, Huh J, Ryu H-Y, Lee Y-H, Kim J-S. Nondegenerate monopole-mode two-dimensional photonic band gap laser. Appl Phys Lett. 2001;79(19):3032-4. https://doi.org/10.1063/1.1416163. Accessed 13 Jan 2021.

115. Ryu H-Y, Kim S-H, Park H-G, Hwang J-K, Lee Y-H, Kim J-S. Square-lattice photonic band-gap single-cell laser operating in the lowest-order whispering gallery mode. Appl Phys Lett. 2002;80(21):3883-5. https://doi.org/10. 1063/1.1480103. Accessed 11 Jan 2021.

116. Srinivasan $\mathrm{K}$, Barclay PE, Painter O, Chen J, Cho AY, Gmachl C. Experimental demonstration of a high quality factor photonic crystal microcavity. Appl Phys Lett. 2003;83(10):1915-7. https://doi.org/10.1063/1. 1606866. Accessed 11 Jan 2021.

117. Nomura M, Iwamoto S, Watanabe K, Kumagai N, Nakata Y, Ishida S, Arakawa Y. Room temperature continuous-wave lasing in photonic crystal nanocavity. Opt Express. 2006;14(13):6308. https://doi.org/10.1364/OE. 14.006308. Accessed 12 Jan 2021.

118. Strauf S, Hennessy K, Rakher MT, Choi Y-S, Badolato A, Andreani LC, Hu EL, Petroff PM, Bouwmeester D. Self-Tuned Quantum Dot Gain in Photonic Crystal Lasers. Phys Rev Lett. 2006;96(12):127404. https://doi.org/10. 1103/PhysRevLett.96.127404. Accessed 11 Jan 2021.

119. Happ TD, Kamp M, Forchel A, Gentner J-L, Goldstein L. Two-dimensional photonic crystal coupled-defect laser diode. Appl Phys Lett. 2003;82(1):4-6. https://doi.org/10.1063/1.1527703. Accessed 11 Jan 2021.

120. Srinivasan K, Painter O. Momentum space design of high-Q photonic crystal optical cavities. Opt Express. 2002;10(15):670. https://doi.org/10.1364/OE.10.000670. Accessed 13 Jan 2021.

121. Vuckovic J, Loncar M, Mabuchi H, Scherer A. Optimization of the $Q$ factor in photonic crystal microcavities. IEEE J Quantum Electron. 2002;38(7):850-6. https://doi.org/10.1109/JQE.2002.1017597. Accessed 13 Jan 2021.

122. Zhang Z, Qiu M. Small-volume waveguide-section high Q microcavities in $2 D$ photonic crystal slabs. Opt Express. 2004;12(17):3988. https://doi.org/10.1364/OPEX.12.003988. Accessed 16 Jan 2021.

123. Chutinan A, Noda S. Waveguides and waveguide bends in two-dimensional photonic crystal slabs. Phys Rev B. 2000;62(7):4488-92. https://doi.org/10.1103/PhysRevB.62.4488.

124. Englund D, Fattal D, Waks E, Solomon G, Zhang B, Nakaoka T, Arakawa Y, Yamamoto Y, Vučković J. Controlling the Spontaneous Emission Rate of Single Quantum Dots in a Two-Dimensional Photonic Crystal. Phys Rev Lett. 2005;95(1):013904. https://doi.org/10.1103/PhysRevLett.95.013904. Accessed 12 Jan 2021.

125. Chang W-H, Chen W-Y, Chang H-S, Hsieh T-P, Chyi J-I, Hsu T-M. Efficient Single-Photon Sources Based on Low-Density Quantum Dots in Photonic-Crystal Nanocavities. Phys Rev Lett. 2006;96(11):117401. https://doi.org/ 10.1103/PhysRevLett.96.117401. Accessed 13 Jan 2021.

126. Englund D, Faraon A, Fushman I, Stoltz N, Petroff P, Vučković J. Controlling cavity reflectivity with a single quantum dot. Nature. 2007;450(7171):857-61. https://doi.org/10.1038/nature06234. Accessed 12 March 2021.

127. Thon SM, Rakher MT, Kim H, Gudat J, Irvine WTM, Petroff PM, Bouwmeester D. Strong coupling through optical positioning of a quantum dot in a photonic crystal cavity. Appl Phys Lett. 2009;94(11):111115. https://doi.org/10. 1063/1.3103885. Accessed 12 March 2021.

128. Bennett AJ, Pooley MA, Stevenson RM, Ward MB, Patel RB, de la Giroday AB, Sköld N, Farrer I, Nicoll CA, Ritchie DA, Shields AJ. Electric-field-induced coherent coupling of the exciton states in a single quantum dot. Nat Phys. 2010;6(12):947-50. https://doi.org/10.1038/nphys1780. Accessed 12 March 2021.

129. Hennessy K, Badolato A, Winger M, Gerace D, Atatüre M, Gulde S, Fält S, Hu EL, Imamoğlu A. Quantum nature of a strongly coupled single quantum dot-cavity system. Nature. 2007:445(7130):896-9. https://doi.org/10.1038/ nature05586. _eprint: 0610034. 
130. Barth M, Nüsse N, Löchel B, Benson O. Controlled coupling of a single-diamond nanocrystal to a photonic crystal cavity. Opt Lett. 2009;34(7):1108. https://doi.org/10.1364/OL.34.001108. Accessed 12 March 2021.

131. Lyasota A, Borghardt S, Jarlov C, Dwir B, Gallo P, Rudra A, Kapon E. Integration of multiple site-controlled pyramidal quantum dot systems with photonic-crystal membrane cavities. J Cryst Growth. 2015;414:192-5. https:// doi.org/10.1016/j.jcrysgro.2014.10.028. Accessed 12 March 2021.

132. Gopinath A, Miyazono E, Faraon A, Rothemund PWK. Engineering and mapping nanocavity emission via precision placement of DNA origami. Nature. 2016;535(7612):401-5. https://doi.org/10.1038/ nature18287. Accessed 08 Nov 2020.

133. Liberal I, Engheta N. Zero-index structures as an alternative platform for quantum optics. Proc Natl Acad Sci. 2017;114(5):822-7. https://doi.org/10.1073/pnas.1611924114.

134. Zhou M, Ying L, Lu L, Shi L, Zi J, Yu Z. Electromagnetic scattering laws in weyl systems. Nat Commun. 2017;8(1):1-7.

135. Ying L, Zhou M, Mattei M, Liu B, Campagnola P, Goldsmith RH, Yu Z. Extended range of dipole-dipole interactions in periodically structured photonic media. Phys Rev Lett. 2019;123:173901. https://doi.org/10.1103/ PhysRevLett.123.173901.

136. García-Elcano I, González-Tudela A, Bravo-Abad J. Tunable and robust long-range coherent interactions between quantum emitters mediated by weyl bound states. Phys Rev Lett. 2020;125:163602. https://doi.org/10.1103/ PhysRevLett.125.163602.

137. Perczel J, Lukin MD. Theory of dipole radiation near a dirac photonic crystal. Phys Rev A. 2020;101:033822. https:// doi.org/10.1103/PhysRevA.101.033822.

138. Veronis G, Dutton RW, Fan S. Metallic photonic crystals with strong broadband absorption at optical frequencies over wide angular range. J Appl Phys. 2005;97(9):093104. https://doi.org/10.1063/1.1889248. Accessed 15 Jan 2021.

139. Barth M, Nüsse N, Stingl J, Löchel B, Benson O. Emission properties of high-Q silicon nitride photonic crystal heterostructure cavities. Appl Phys Lett. 2008;93:021112. https://doi.org/10.1063/1.2958346.

140. Rivoire K, Faraon A, Vučković J. Gallium phosphide photonic crystal nanocavities in the visible. Appl Phys Lett. 2008;93:063103. https://doi.org/10.1063/1.2971200.

141. Hu X, Jiang P, Ding C, Yang H, Gong Q. Picosecond and low-power all-optical switching based on an organic photonic-bandgap microcavity. Nat Photonics. 2008;2(3):185-9. https://doi.org/10.1038/nphoton.2007. 299. Accessed 16 Nov 2020.

142. Gan X, Gao Y, Fai Mak K, Yao X, Shiue R-J, van der Zande A, Trusheim ME, Hatami F, Heinz TF, Hone J, Englund D. Controlling the spontaneous emission rate of monolayer MoS2 in a photonic crystal nanocavity. Appl Phys Lett. 2013;103(18):181119. https://doi.org/10.1063/1.4826679. Accessed 18 March 2021.

143. Gan X, Mak KF, Gao Y, You Y, Hatami F, Hone J, Heinz TF, Englund D. Strong Enhancement of Light-Matter Interaction in Graphene Coupled to a Photonic Crystal Nanocavity. Nano Lett. 2012;12(11):5626-31. https://doi. org/10.1021/nl302746n. Accessed 18 March 2021.

144. Zuo Y, Yu W, Liu C, Cheng X, Qiao R, Liang J, Zhou X, Wang J, Wu M, Zhao Y, Gao P, Wu S, Sun Z, Liu K, Bai X, Liu Z. Optical fibres with embedded two-dimensional materials for ultrahigh nonlinearity. Nat Nanotechnol. 2020;15(12):987-91. https://doi.org/10.1038/s41565-020-0770-x. Accessed 18 March 2021.

145. Thyrrestrup H, Sapienza L, Lodahl P. Extraction of the $\beta$-factor for single quantum dots coupled to a photonic crystal waveguide. Appl Phys Lett. 2010;96(23):231106. https://doi.org/10.1063/1.3446873. Accessed 15 March 2021.

146. Fleming JG, Lin S-Y. Three-dimensional photonic crystal with a stop band from 1.35 to $1.95 \mathrm{um}$. Opt Lett. 1999;24(1):49. https://doi.org/10.1364/OL.24.000049. Accessed 16 Jan 2021.

147. Leistikow MD, Mosk AP, Yeganegi E, Huisman SR, Lagendijk A, Vos WL. Inhibited Spontaneous Emission of Quantum Dots Observed in a 3D Photonic Band Gap. Phys Rev Lett. 2011;107(19):193903. https://doi.org/10.1103/ PhysRevLett.107.193903. Accessed 15 Jan 2021.

148. Zhang F, Ren J, Shan L, Duan X, Li Y, Zhang T, Gong Q, Gu Y. Chiral cavity quantum electrodynamics with coupled nanophotonic structures. Phys Rev A. 2019;100(5):053841. https://doi.org/10.1103/PhysRevA.100. 053841. Publisher: American Physical Society.

149. Notomi M, Yamada K, Shinya A, Takahashi J, Takahashi C, Yokohama I. Extremely Large Group-Velocity Dispersion of Line-Defect Waveguides in Photonic Crystal Slabs. Phys Rev Lett. 2001;87(25):253902. https://doi.org/10.1103/ PhysRevLett.87.253902. Accessed 16 March 2021.

150. Lin SY, Fleming JG, Li ZY, El-Kady I, Biswas R, Ho KM. Origin of absorption enhancement in a tungsten, three-dimensional photonic crystal. J Opt Soc Am B. 2003;20(7):1538. https://doi.org/10.1364/JOSAB.20. 001538. Accessed 15 Jan 2021.

151. Javadi A, Söllner I, Arcari M, Hansen SL, Midolo L, Mahmoodian S, Kiršansk- G, Pregnolato T, Lee EH, Song JD, Stobbe S, Lodahl P. Single-photon non-linear optics with a quantum dot in a waveguide. Nat Commun. 2015;6: 8655. https://doi.org/10.1038/ncomms9655.

152. Miroshnichenko AE, Flach S, Kivshar YS. Fano resonances in nanoscale structures. Rev Mod Phys. 2010;82(3): 2257-98. https://doi.org/10.1103/RevModPhys.82.2257. _eprint: 0902.3014.

153. Zhou W, Zhao D, Shuai Y-C, Yang H, Chuwongin S, Chadha A, Seo J-H, Wang KX, Liu V, Ma Z, Fan S. Progress in 2D photonic crystal Fano resonance photonics. Prog Quantum Electron. 2014;38(1):1-74. https://doi.org/10.1016/j. pquantelec.2014.01.001.

154. Fan S, Suh W, Joannopoulos JD. Temporal coupled-mode theory for the Fano resonance in optical resonators. J Opt Soc Am A. 2003;20(3):569-72.

155. Ding W, Luk'yanchuk B, Qiu C-W. Ultrahigh-contrast-ratio silicon Fano diode. Phys Rev A. 2012;85(2):025806. https://doi.org/10.1103/PhysRevA.85.025806. Accessed 17 March 2021.

156. Yang H, Zhao D, Chuwongin S, Seo J-H, Yang W, Shuai Y, Berggren J, Hammar M, Ma Z, Zhou W. Transfer-printed stacked nanomembrane lasers on silicon. Nat Photonics. 2012;6(9):615-20. https://doi.org/10. 1038/nphoton.2012.160. Accessed 15 Jan 2021. 
157. Chai Z, Hu X, Gong Q. All-optical switching based on a tunable Fano-like resonance in nonlinear ferroelectric photonic crystals. J Opt. 2013;15:085001. https://doi.org/10.1088/2040-8978/15/8/085001.

158. Xie J, Hu X, Li C, Wang F, Xu P, Tong L, Yang H, Gong Q. On-Chip Dual Electro-Optic and Optoelectric Modulation Based on ZnO Nanowire-Coated Photonic Crystal Nanocavity. Adv Opt Mater. 2018;6(17):1800374. https://doi.org/10.1002/adom.201800374. Accessed 15 Jan 2021.

159. Della Villa A, Enoch S, Tayeb G, Pierro V, Galdi V, Capolino F. Band Gap Formation and Multiple Scattering in Photonic Quasicrystals with a Penrose-Type Lattice. Phys Rev Lett. 2005;94(18):183903. https://doi.org/10.1103/ PhysRevLett.94.183903. Accessed 15 Jan 2021.

160. Jin C, Meng X, Cheng B, Li Z, Zhang D. Photonic gap in amorphous photonic materials. Phys Rev B. 2001;63(19): 195107. https://doi.org/10.1103/PhysRevB.63.195107. Accessed 17 March 2021.

161. Notomi M, Suzuki H, Tamamura T, Edagawa K. Lasing Action due to the Two-Dimensional Quasiperiodicity of Photonic Quasicrystals with a Penrose Lattice. Phys Rev Lett. 2004;92(12):123906. https://doi.org/10.1103/ PhysRevLett.92.123906. Accessed 15 Jan 2021.

162. Lodahl P, Floris van Driel A, Nikolaev IS, Irman A, Overgaag K, Vanmaekelbergh D, Vos WL. Controlling the dynamics of spontaneous emission from quantum dots by photonic crystals. Nature. 2004;430(7000):654-7. https://doi.org/10.1038/nature02772. Accessed 15 Jan 2021.

163. Arcari M, Söllner I, Javadi A, Lindskov Hansen S, Mahmoodian S, Liu J, Thyrrestrup H, Lee EH, Song JD, Stobbe S, Lodahl P. Near-Unity Coupling Efficiency of a Quantum Emitter to a Photonic Crystal Waveguide. Phys Rev Lett. 2014;113(9):093603. https://doi.org/10.1103/PhysRevLett.113.093603. Accessed 15 March 2021.

164. Noda S, Yokoyama M, Masahiro I, Alongkarn C, Mochisuki M. Polarization Mode Control of Two-Dimensional Photonic Crystal Laser by Unit Cell Structure Design. Science. 2001;293(5532):1123-5. https://doi.org/10.1126/ science.1061738. Accessed 11 Jan 2021.

165. Zhou Y-S, Wang X-H, Gu B-Y, Wang F-H. Switching Control of Spontaneous Emission by Polarized Atoms in Two-Dimensional Photonic Crystals. Phys Rev Lett. 2006;96(10):103601. https://doi.org/10.1103/PhysRevLett.96. 103601. Accessed 15 Jan 2021.

166. Noda S, Fujita M, Asano T. Spontaneous-emission control by photonic crystals and nanocavities. Nat Photonics. 2007;1 (8):449-58. https://doi.org/10.1038/nphoton.2007.141. Accessed 12 Jan 2021.

167. Lecamp G, Lalanne P, Hugonin JP. Very Large Spontaneous-Emission $\beta$ Factors in Photonic-Crystal Waveguides. Phys Rev Lett. 2007;99(2):023902. https://doi.org/10.1103/PhysRevLett.99.023902. Accessed 14 Jan 2021.

168. Banaee MG, Pattantyus-Abraham AG, McCutcheon MW, Rieger GW, Young JF. Efficient coupling of photonic crystal microcavity modes to a ridge waveguide. Appl Phys Lett. 2007;90(19):193106. https://doi.org/10.1063/1. 2737369. Accessed 15 March 2021.

169. Bliokh KY, Rodríguez-Fortuño FJ, Nori F, Zayats AV. Spin-orbit interactions of light. Nat Photonics. 2015;9(12): 796-808. https://doi.org/10.1038/nphoton.2015.201. ISBN: doi:10.1038/nphoton.2015.201_eprint: 1505.02864

170. Lodahl P, Mahmoodian S, Stobbe S, Rauschenbeutel A, Schneeweiss P, Volz J, Pichler H, Zoller P. Chiral quantum optics. Nature. 2017;541(7638):473-80. https://doi.org/10.1038/nature21037. ISBN: 1476-4687 (Electronic)\$ \backslash\$r0028-0836 (Linking) Publisher: Nature Publishing Group_eprint: 1608.00446.

171. Söllner I, Mahmoodian S, Hansen SL, Midolo L, Javadi A, Kiršanskè G, Pregnolato T, El-Ella H, Lee EH, Song JD, Stobbe S, Lodahl P. Deterministic photon-emitter coupling in chiral photonic circuits. Nat Nanotechnol. 2015;10(9):775-8. https://doi.org/10.1038/nnano.2015.159. Accessed 15 Jan 2021.

172. Yoshie T, Scherer A, Hendrickson J, Khitrova G, Gibbs HM, Rupper G, Ell C, Shchekin OB, Deppe DG. Vacuum Rabi splitting with a single quantum dot in a photonic crystal nanocavity. Nature. 2004;432(7014):200-3. https:// doi.org/10.1038/nature03119. Accessed 16 Oct 2020.

173. Faraon A, Fushman I, Englund D, Stoltz N, Petroff P, Vučković J. Coherent generation of non-classical light on a chip via photon-induced tunnelling and blockade. Nat Phys. 2008;4(11):859-63. https://doi.org/10.1038/nphys1078.

174. Fushman I, Englund D, Faraon A, Stoltz N, Petroff P, Vučković J. Controlled Phase Shifts with a Single Quantum Dot. Science. 2008;320(5877):769-72. https://doi.org/10.1126/science.1154643. Accessed 19 Oct 2020.

175. Bose R, Sridharan D, Kim H, Solomon GS, Waks E. Low-Photon-Number Optical Switching with a Single Quantum Dot Coupled to a Photonic Crystal Cavity. Phys Rev Lett. 2012;108(22):227402. https://doi.org/10.1103/PhysRevLett. 108.227402. Accessed 08 Jan 2021.

176. Englund D, Majumdar A, Bajcsy M, Faraon A, Petroff P, Vučković J. Ultrafast Photon-Photon Interaction in a Strongly Coupled Quantum Dot-Cavity System. Phys Rev Lett. 2012;108(9):093604. https://doi.org/10.1103/ PhysRevLett.108.093604. Accessed 08 Jan 2021.

177. Reinhard A, Volz T, Winger M, Badolato A, Hennessy KJ, Hu EL, Imamoğlu A. Strongly correlated photons on a chip. Nat Photonics. 2012;6(2):93-6. https://doi.org/10.1038/nphoton.2011.321. _eprint: 1108.3053.

178. Müller K, Rundquist A, Fischer KA, Sarmiento T, Lagoudakis KG, Kelaita YA, Sánchez Muñoz C, Del Valle E, Laussy FP, Vučković J. Coherent generation of nonclassical light on chip via detuned photon blockade. Phys Rev Lett. 2015;114(23):233601. https://doi.org/10.1103/PhysRevLett.114.233601._eprint: 1408.5942.

179. Sommerfeld A. Über die fortpflanzung electrodynamischer wellen längs eines drahtes. Ann Phys und Chemie. 1899;67:233-90.

180. Wood RW. Xlii. on a remarkable case of uneven distribution of light in a diffraction grating spectrum. Lond Edinb Dublin Philos Mag J Sci. 1902;4(21):396-402. https://doi.org/10.1080/14786440209462857.

181. Zenneck J. Über die fortpflanzung ebener elektromagnetischer wellen längs einer ebenen leiterfläche und ihre beziehung zur drahtlosen telegraphie. Ann d Phys. 1907;23:846-66.

182. Ritchie RH. Plasma losses by fast electrons in thin films. Phys Rev. 1957;106:874-81. https://doi.org/10.1103/ PhysRev.106.874.

183. Ritchie RH, Arakawa ET, Cowan JJ, Hamm RN. Surface-plasmon resonance effect in grating diffraction. Phys Rev Lett. 1968;21:1530-3. https://doi.org/10.1103/PhysRevLett.21.1530.

184. Kretschmann E, Raether H. Notizen: Radiative decay of non radiative surface plasmons excited by light. Z für Naturforsh A. 1968;23(12):2135-6. https://doi.org/10.1515/zna-1968-1247. 
185. Raether H. Surface Plasmons on Smooth and Rough Surfaces and on Gratings. Heidelberg: Springer; 1988.

186. Pigeon F, Salakhutdinov IF, Tishchenko AV. Identity of long-range surface plasmons along asymmetric structures and their potential for refractometric sensors. J Appl Phys. 2001;90(2):852-9. https://doi.org/10.1063/1.1380407.

187. Burke JJ, Stegeman GI, Tamir T. Surface-polariton-like waves guided by thin, lossy metal films. Phys Rev B. 1986;33: 5186-201. https://doi.org/10.1103/PhysRevB.33.5186.

188. Zayats AV, Smolyaninov II, Maradudin AA. Nano-optics of surface plasmon polaritons. Phys Rep. 2005;408(3): 131-314. https://doi.org/10.1016/j.physrep.2004.11.001.

189. Ozbay E. Plasmonics: Merging photonics and electronics at nanoscale dimensions. Science. 2006;311(5758): 189-93. https://doi.org/10.1126/science.1114849.

190. Lalanne P, Hugonin JP, Liu HT, Wang B. A microscopic view of the electromagnetic properties of sub- $\lambda$ metallic surfaces. Surf Sci Rep. 2009;64(10):453-69. https://doi.org/10.1016/j.surfrep.2009.07.003.

191. Mansuripur M, Zakharian AR, Moloney JV. Surface plasmon polaritons on metallic surfaces. Opt Photon News. 2007;18(4):44-9. https://doi.org/10.1364/OPN.18.4.000044.

192. Kelly KL, Coronado E, Zhao LL, Schatz GC. The optical properties of metal nanoparticles: The influence of size, shape, and dielectric environment. J Phys Chem B. 2003;107(3):668-77. https://doi.org/10.1021/jp026731y.

193. Huffman CFBDR. Absorption and Scattering of Light by Small Particles. Weinheim: Wiley-VCH Verlag GmbH \& Co. KGaA; 1998.

194. Kreibig U, Vollmer M. Optical Properties of Metal Clusters. Berlin Heidelberg: Springer; 1995.

195. Taminiau T, Stefani F, Segerink FB, Van Hulst N. Optical antennas direct single-molecule emission. Nat Photonics. 2008;2(4):234-7.

196. Johnson PB, Christy RW. Optical constants of the noble metals. Phys Rev B. 1972;6:4370-9. https://doi.org/10.1103/ PhysRevB.6.4370.

197. Bozhevolnyi SI, Volkov VS, Devaux E, Laluet J-Y, Ebbesen TW. Channel plasmon subwavelength waveguide components including interferometers and ring resonators. Nature. 2006;440(7083):508-11.

198. Wiley BJ, Chen Y, McLellan JM, Xiong Y, Li Z-Y, Ginger D, Xia Y. Synthesis and optical properties of silver nanobars and nanorice. Nano Lett. 2007;7(4):1032-6. https://doi.org/10.1021/nl070214f. PMID: 17343425.

199. Ditlbacher H, Hohenau A, Wagner D, Kreibig U, Rogers M, Hofer F, Aussenegg FR, Krenn JR. Silver nanowires as surface plasmon resonators. Phys Rev Lett. 2005;95:257403. https://doi.org/10.1103/PhysRevLett.95.257403.

200. Wang $P$, Zhang $L$, Xia Y, Tong $L$, Xu X, Ying Y. Polymer nanofibers embedded with aligned gold nanorods: A new platform for plasmonic studies and optical sensing. Nano Lett. 2012;12(6):3145-50. https://doi.org/10.1021/ nl301055f. PMID: 22582809.

201. Shan X, Díez-Pérez I, Wang L, Wiktor P, Gu Y, Zhang L, Wang W, Lu J, Wang S, Gong Q, et al. Imaging the electrocatalytic activity of single nanoparticles. Nat Nanotechnol. 2012;7(10):668-72.

202. Peng-Fei Y, Ying G, Qi-Huang G. Surface plasmon polariton and mode transformation in a nanoscale lossy metallic cylindrical cable. Chin Phys B. 2008;17(10):3880-93. https://doi.org/10.1088/1674-1056/17/10/055.

203. Wang L, Gu Y, Hu X, Gong Q. Long-range surface plasmon polariton modes with a large field localized in a nanoscale gap. Appl Phys B. 2011;104(4):919-24.

204. Chen X-W, Sandoghdar V, Agio M. Highly efficient interfacing of guided plasmons and photons in nanowires. Nano Lett. 2009;9(11):3756-61. https://doi.org/10.1021/nl9019424. PMID: 19754143.

205. Chen J, Li Z, Yue S, Xiao J, Gong Q. Plasmon-induced transparency in asymmetric t-shape single slit. Nano Lett. 2012;12(5):2494-8. https://doi.org/10.1021/nl300659v. PMID: 22471626.

206. Liu M, Lee T-W, Gray SK, Guyot-Sionnest P, Pelton M. Excitation of dark plasmons in metal nanoparticles by a localized emitter. Phys Rev Lett. 2009;102:107401. https://doi.org/10.1103/PhysRevLett.102.107401.

207. Zhang W, Govorov AO, Bryant GW. Semiconductor-metal nanoparticle molecules: Hybrid excitons and the nonlinear fano effect. Phys Rev Lett. 2006;97:146804. https://doi.org/10.1103/PhysRevLett.97.146804.

208. Zhang W, Govorov AO. Quantum theory of the nonlinear fano effect in hybrid metal-semiconductor nanostructures: The case of strong nonlinearity. Phys Rev B. 2011;84:081405. https://doi.org/10.1103/PhysRevB.84 081405.

209. Chang DE, Sørensen AS, Hemmer PR, Lukin MD. Quantum optics with surface plasmons. Phys Rev Lett. 2006;97: 053002. https://doi.org/10.1103/PhysRevLett.97.053002.

210. Berini P, De Leon I. Surface plasmon-polariton amplifiers and lasers. Nat Photonics. 2012;6(1):16-24.

211. Chen J, Gan F, Wang Y, Li G. Plasmonic sensing and modulation based on fano resonances. Adv Opt Mater. 2018;6(9):1701152. https://doi.org/10.1002/adom.201701152.

212. Li H, Huang Y, Hou G, Xiao A, Chen P, Liang H, Huang Y, Zhao X, Liang L, Feng X, Guan B-O. Single-molecule detection of biomarker and localized cellular photothermal therapy using an optical microfiber with nanointerface. Sci Adv. 2019;5(12):eaax4659. https://doi.org/10.1126/sciadv.aax4659.

213. Sigle DO, Hugall JT, Ithurria S, Dubertret B, Baumberg JJ. Probing confined phonon modes in individual cdse nanoplatelets using surface-enhanced raman scattering. Phys Rev Lett. 2014;113:087402. https://doi.org/10.1103/ PhysRevLett.113.087402.

214. Chen $X$, Chen Y, Yan M, Qiu M. Nanosecond photothermal effects in plasmonic nanostructures. ACS Nano. 2012;6(3):2550-7. https://doi.org/10.1021/nn2050032. PMID: 22356648.

215. Han B, Gao X, Shi L, Zheng Y, Hou K, Lv J, Guo J, Zhang W, Tang Z. Geometry-modulated magnetoplasmonic optical activity of au nanorod-based nanostructures. Nano Lett. 2017;17(10):6083-9. https://doi.org/10.1021/acs. nanolett.7b02583. PMID: 28953401.

216. Cai Y-J, Li M, Ren X-F, Zou C-L, Xiong X, Lei H-L, Liu B-H, Guo G-P, Guo G-C. High-visibility on-chip quantum interference of single surface plasmons. Phys Rev Appl. 2014;2:014004. https://doi.org/10.1103/PhysRevApplied.2. 014004.

217. Li M, Zou C-L, Ren X-F, Xiong X, Cai Y-J, Guo G-P, Tong L-M, Guo G-C. Transmission of photonic quantum polarization entanglement in a nanoscale hybrid plasmonic waveguide. Nano Lett. 2015;15(4):2380-4. https://doi. org/10.1021/nl504636x. PMID: 25775140.

218. Rong K, Gan F, Shi K, Chu S, Chen J. Configurable integration of on-chip quantum dot lasers and subwavelength plasmonic waveguides. Adv Mater. 2018;30(21):1706546. https://doi.org/10.1002/adma.201706546. 
219. Peng P, Liu Y-C, Xu D, Cao Q-T, Lu G, Gong Q, Xiao Y-F. Enhancing coherent light-matter interactions through microcavity-engineered plasmonic resonances. Phys Rev Lett. 2017;119:233901. https://doi.org/10.1103/ PhysRevLett.119.233901.

220. Wei H, Pan D, Zhang S, Li Z, Li Q, Liu N, Wang W, Xu H. Plasmon waveguiding in nanowires. Chem Rev. 2018;118(6):2882-926. https://doi.org/10.1021/acs.chemrev.7b00441. PMID: 29446301.

221. Xu D, Xiong X, Wu L, Ren X-F, Png CE, Guo G-C, Gong Q, Xiao Y-F. Quantum plasmonics: new opportunity in fundamental and applied photonics. Adv Opt Photon. 2018;10(4):703-56. https://doi.org/10.1364/AOP.10.000703.

222. Waks E, Sridharan D. Cavity qed treatment of interactions between a metal nanoparticle and a dipole emitter. Phys Rev A. 2010;82:043845. https://doi.org/10.1103/PhysRevA.82.043845.

223. Anger P, Bharadwaj $P$, Novotny L. Enhancement and quenching of single-molecule fluorescence. Phys Rev Lett. 2006;96:113002. https://doi.org/10.1103/PhysRevLett.96.113002.

224. Akimov A, Mukherjee A, Yu C, Chang D, Zibrov A, Hemmer P, Park H, Lukin M. Generation of single optical plasmons in metallic nanowires coupled to quantum dots. Nature. 2007;450(7168):402-6.

225. Ruppin R. Decay of an excited molecule near a small metal sphere. J Chem Phys. 1982;76(4):1681-4. https://doi. org/10.1063/1.443196.

226. Kramer A, Trabesinger W, Hecht B, Wild UP. Optical near-field enhancement at a metal tip probed by a single fluorophore. Appl Phys Lett. 2002;80(9):1652-4. https://doi.org/10.1063/1.1453479.

227. Dulkeith E, Ringler M, Klar TA, Feldmann J, Muñoz Javier A, Parak WJ. Gold nanoparticles quench fluorescence by phase induced radiative rate suppression. Nano Lett. 2005;5(4):585-9. https://doi.org/10.1021/nl0480969. PMID: 15826091.

228. Trabesinger W, Kramer A, Kreiter M, Hecht B, Wild UP. Single-molecule near-field optical energy transfer microscopy. Appl Phys Lett. 2002;81(11):2118-20. https://doi.org/10.1063/1.1506952.

229. Esteban R, Teperik TV, Greffet JJ. Optical patch antennas for single photon emission using surface plasmon resonances. Phys Rev Lett. 2010;104:026802. https://doi.org/10.1103/PhysRevLett.104.026802.

230. Van Vlack C, Kristensen PT, Hughes S. Spontaneous emission spectra and quantum light-matter interactions from a strongly coupled quantum dot metal-nanoparticle system. Phys Rev B. 2012;85:075303. https://doi.org/10.1103/ PhysRevB.85.075303.

231. Chen Y, Nielsen TR, Gregersen N, Lodahl P, Mørk J. Finite-element modeling of spontaneous emission of a quantum emitter at nanoscale proximity to plasmonic waveguides. Phys Rev B. 2010;81:125431. https://doi.org/10. 1103/PhysRevB.81.125431.

232. Gonzalez-Tudela A, Rodríguez FJ, Quiroga L, Tejedor C. Dissipative dynamics of a solid-state qubit coupled to surface plasmons: From non-markov to markov regimes. Phys Rev B. 2010;82:115334. https://doi.org/10.1103/ PhysRevB.82.115334.

233. Lee K, Chen X, Eghlidi H, Kukura P, Lettow R, Renn A, Sandoghdar V, Götzinger S. A planar dielectric antenna for directional single-photon emission and near-unity collection efficiency. Nat Photonics. 2011;5(3):166-9.

234. Chu X-L, Brenner TJK, Chen X-W, Ghosh Y, Hollingsworth JA, Sandoghdar V, Götzinger S. Experimental realization of an optical antenna designed for collecting $99 \%$ of photons from a quantum emitter. Optica. 2014;1(4):203-8. https://doi.org/10.1364/OPTICA.1.000203.

235. Pfab RJ, Zimmermann J, Hettich C, Gerhardt I, Renn A, Sandoghdar V. Aligned terrylene molecules in a spin-coated ultrathin crystalline film of p-terphenyl. Chem Phys Lett. 2004;387(4):490-5. https://doi.org/10.1016/j. cplett.2004.02.040.

236. Liaw J. Analysis of a bowtie nanoantenna for the enhancement of spontaneous emission. IEEE J Sel Top Quant Electron. 2008;14(6):1441-7. https://doi.org/10.1109/JSTQE.2008.916755.

237. Lee K-G, Eghlidi H, Chen X-W, Renn A, Götzinger S, Sandoghdar V. Spontaneous emission enhancement of a single molecule by a double-sphere nanoantenna across an interface. Opt Express. 2012;20(21):23331-8. https:// doi.org/10.1364/OE.20.023331.

238. Qian Z, Li Z, Hao H, Shan L, Zhang Q, Dong J, Gong Q, Gu Y. Absorption reduction of large purcell enhancement enabled by topological state-led mode coupling. Phys Rev Lett. 2021;126:023901. https://doi.org/10.1103/ PhysRevLett.126.023901.

239. Guo X, Qiu M, Bao J, Wiley BJ, Yang Q, Zhang X, Ma Y, Yu H, Tong L. Direct coupling of plasmonic and photonic nanowires for hybrid nanophotonic components and circuits. Nano Lett. 2009;9(12):4515-9. https://doi.org/10. 1021/nl902860d. PMID: 19995088.

240. Jun YC, Kekatpure RD, White JS, Brongersma ML. Nonresonant enhancement of spontaneous emission in metal-dielectric-metal plasmon waveguide structures. Phys Rev B. 2008;78:153111. https://doi.org/10.1103/ PhysRevB.78.153111.

241. Lassiter JB, MCGuire F, Mock JJ, Ciracì C, Hill RT, Wiley BJ, Chilkoti A, Smith DR. Plasmonic waveguide modes of film-coupled metallic nanocubes. Nano Lett. 2013;13(12):5866-72. https://doi.org/10.1021/n/402660s. PMID: 24199752.

242. Le F, Lwin NZ, Steele JM, Käll M, Halas NJ, Nordlander P. Plasmons in the metallic nanoparticle-film system as a tunable impurity problem. Nano Lett. 2005;5(10):2009-13. https://doi.org/10.1021/nl0515100. PMID: 16218728.

243. Lévêque G, Martin OJF. Optical interactions in a plasmonic particle coupled to a metallic film. Opt Express. 2006;14(21):9971-81. https://doi.org/10.1364/OE.14.009971.

244. Mock JJ, Hill RT, Degiron A, Zauscher S, Chilkoti A, Smith DR. Distance-dependent plasmon resonant coupling between a gold nanoparticle and gold film. Nano Lett. 2008;8(8):2245-52. https://doi.org/10.1021/ nl080872f. PMID: 18590340.

245. Russell KJ, Liu T-L, Cui S, Hu EL. Large spontaneous emission enhancement in plasmonic nanocavities. Nat Photonics. 2012;6(7):459-62.

246. Akselrod GM, Argyropoulos C, Hoang TB, Ciracì C, Fang C, Huang J, Smith DR, Mikkelsen MH. Probing the mechanisms of large purcell enhancement in plasmonic nanoantennas. Nat Photonics. 2014;8(11):835-40

247. Zhang G, Jia S, Gu Y, Chen J. Brightening and guiding single-photon emission by plasmonic waveguide-slit structures on a metallic substrate. Laser Photonics Rev. 2019;13(10):1900025. https://doi.org/10.1002/lpor. 201900025. 
248. Tserkezis C, Esteban R, Sigle DO, Mertens J, Herrmann LO, Baumberg JJ, Aizpurua J. Hybridization of plasmonic antenna and cavity modes: Extreme optics of nanoparticle-on-mirror nanogaps. Phys Rev A. 2015;92:053811. https://doi.org/10.1103/PhysRevA.92.053811.

249. Yang G, Shen Q, Niu Y, Wei H, Bai B, Mikkelsen MH, Sun H-B. Unidirectional, ultrafast, and bright spontaneous emission source enabled by a hybrid plasmonic nanoantenna. Laser Photonics Rev. 2020;14(3):1900213. https:// doi.org/10.1002/lpor.201900213.

250. Yang Y, Zhen B, Hsu CW, Miller OD, Joannopoulos JD, Soljačić M. Optically thin metallic films for high-radiative-efficiency plasmonics. Nano Lett. 2016;16(7):4110-7. https://doi.org/10.1021/acs.nanolett. 6b00853. PMID: 27244596.

251. Hao H, Ren J, Duan X, Lu G, Khoo IC, Gong Q, Gu Y. High-contrast switching and high-efficiency extracting for spontaneous emission based on tunable gap surface plasmon. Sci Rep. 2018;8(1):1-11.

252. Chen X-W, Agio M, Sandoghdar V. Metallodielectric hybrid antennas for ultrastrong enhancement of spontaneous emission. Phys Rev Lett. 2012;108:233001. https://doi.org/10.1103/PhysRevLett.108.233001.

253. Yang G, Niu Y, Wei H, Bai B, Sun H-B. Greatly amplified spontaneous emission of colloidal quantum dots mediated by a dielectric-plasmonic hybrid nanoantenna. Nanophotonics. 2019;8(12):2313-9. https://doi.org/10. 1515/nanoph-2019-0332.

254. Oulton RF, Sorger VJ, Zentgraf T, Ma R-M, Gladden C, Dai L, Bartal G, Zhang X. Plasmon lasers at deep subwavelength scale. Nature. 2009;461(7264):629-32.

255. Hill MT, Marell M, Leong ESP, Smalbrugge B, Zhu Y, Sun M, van Veldhoven PJ, Geluk EJ, Karouta F, Oei Y-S, Nötzel R, Ning C-Z, Smit MK. Lasing in metal-insulator-metal sub-wavelength plasmonic waveguides. Opt Express. 2009;17(13):11107-12. https://doi.org/10.1364/OE.17.011107.

256. Wu X, Xiao Y, Meng C, Zhang X, Yu S, Wang Y, Yang C, Guo X, Ning CZ, Tong L. Hybrid photon-plasmon nanowire lasers. Nano Lett. 2013;13(11):5654-9. https://doi.org/10.1021/nl403325j. PMID: 24144390.

257. Wang S, Wang X-Y, Li B, Chen H-Z, Wang Y-L, Dai L, Oulton RF, Ma R-M. Unusual scaling laws for plasmonic nanolasers beyond the diffraction limit. Nat Commun. 2017;8(1):1-8.

258. Wang Y, Yu J, Mao Y-F, Chen J, Wang S, Chen H-Z, Zhang Y, Wang S-Y, Chen X, Li T, et al. Stable, high-performance sodium-based plasmonic devices in the near infrared. Nature. 2020;581(7809):401-s5.

259. Ma R-M, Oulton RF. Applications of nanolasers. Nat Nanotechnol. 2019;14(1):12-22.

260. Bergman DJ, Stockman MI. Surface plasmon amplification by stimulated emission of radiation: Quantum generation of coherent surface plasmons in nanosystems. Phys Rev Lett. 2003;90:027402. https://doi.org/10.1103/ PhysRevLett.90.027402.

261. Noginov M, Zhu G, Belgrave A, Bakker R, Shalaev V, Narimanov E, Stout S, Herz E, Suteewong T, Wiesner U. Demonstration of a spaser-based nanolaser. Nature. 2009;460(7259):1110-2.

262. Chen H-Z, Hu J-Q, Wang S, Li B, Wang X-Y, Wang Y-L, Dai L, Ma R-M. Imaging the dark emission of spasers. Sci Adv. 2017;3(4):e1601962. https://doi.org/10.1126/sciadv.1601962.

263. Lu Y-J, Kim J, Chen H-Y, Wu C, Dabidian N, Sanders CE, Wang C-Y, Lu M-Y, Li B-H, Qiu X, Chang W-H, Chen L-J, Shvets G, Shih C-K, Gwo S. Plasmonic nanolaser using epitaxially grown silver film. Science. 2012;337(6093):450-3. https://doi.org/10.1126/science.1223504.

264. Ma R-M. Lasing under ultralow pumping. Nat Mater. 2019;18(11):1152-3.

265. Fernandez-Bravo A, Wang D, Barnard ES, Teitelboim A, Tajon C, Guan J, Schatz GC, Cohen BE, Chan EM, Schuck PJ, et al. Ultralow-threshold, continuous-wave upconverting lasing from subwavelength plasmons. Nat Mater. 2019;18(11):1172-6.

266. Ma R-M, Ota S, Li Y, Yang S, Zhang X. Explosives detection in a lasing plasmon nanocavity. Nat Nanotechnol. 2014;9(8):600.

267. Bellessa J, Bonnand C, Plenet JC, Mugnier J. Strong coupling between surface plasmons and excitons in an organic semiconductor. Phys Rev Lett. 2004;93:036404. https://doi.org/10.1103/PhysRevLett.93.036404.

268. Gómez DE, Vernon KC, Mulvaney P, Davis TJ. Surface plasmon mediated strong exciton-photon coupling in semiconductor nanocrystals. Nano Lett. 2010;10(1):274-8. https://doi.org/10.1021/nl903455z. PMID: 20000744.

269. Savasta S, Saija R, Ridolfo A, Di Stefano O, Denti P, Borghese F. Nanopolaritons: Vacuum rabi splitting with a single quantum dot in the center of a dimer nanoantenna. ACS Nano. 2010;4(11):6369-76. https://doi.org/10.1021/ nn100585h. PMID: 21028780.

270. Schlather AE, Large N, Urban AS, Nordlander P, Halas NJ. Near-field mediated plexcitonic coupling and giant rabi splitting in individual metallic dimers. Nano Lett. 2013;13(7):3281-6. https://doi.org/10.1021/nl4014887. PMID: 23746061.

271. Zengin G, Wersäll M, Nilsson S, Antosiewicz TJ, Käll M, Shegai T. Realizing strong light-matter interactions between single-nanoparticle plasmons and molecular excitons at ambient conditions. Phys Rev Lett. 2015;114: 157401. https://doi.org/10.1103/PhysRevLett.114.157401.

272. Liu R, Zhou Z-K, Yu Y-C, Zhang T, Wang H, Liu G, Wei Y, Chen H, Wang X-H. Strong light-matter interactions in single open plasmonic nanocavities at the quantum optics limit. Phys Rev Lett. 2017;1 18:237401. https://doi.org/ 10.1103/PhysRevLett.118.237401.

273. Shang Q, Zhang S, Liu Z, Chen J, Yang P, Li C, Li W, Zhang Y, Xiong Q, Liu X, Zhang Q. Surface plasmon enhanced strong exciton-photon coupling in hybrid inorganic-organic perovskite nanowires. Nano Lett. 2018;18(6):3335-43. https://doi.org/10.1021/acs.nanolett.7b04847. PMID: 29722986.

274. Wu F, Guo J, Huang Y, Liang K, Jin L, Li J, Deng X, Jiao R, Liu Y, Zhang J, Zhang W, Yu L. Plexcitonic optical chirality: Strong exciton-plasmon coupling in chiral j-aggregate-metal nanoparticle complexes. ACS Nano. 2021;15(2):2292-300. https://doi.org/10.1021/acsnano.0c08274. PMID: 33356158.

275. Qian Z, Ren J, Zhang F, Duan X, Gong Q, Gu Y. Nanoscale quantum plasmon sensing based on strong photon-exciton coupling. Nanotechnology. 2020;31(12):125001. https://doi.org/10.1088/1361-6528/ab5dd0.

276. Ren J, Hao H, Qian Z, Duan X, Zhang F, Zhang T, Gong Q, Gu Y. High-dielectric constant enhanced photon-exciton coupling in an evanescent vacuum. J Opt Soc Am B. 2018;35(6):1475-81. https://doi.org/10.1364/ JOSAB.35.001475. 
277. Trügler A, Hohenester U. Strong coupling between a metallic nanoparticle and a single molecule. Phys Rev B. 2008;77:115403. https://doi.org/10.1103/PhysRevB.77.115403.

278. Hakala TK, Toppari JJ, Kuzyk A, Pettersson M, Tikkanen H, Kunttu H, Törmä P. Vacuum rabi splitting and strong-coupling dynamics for surface-plasmon polaritons and rhodamine $6 \mathrm{~g}$ molecules. Phys Rev Lett. 2009;103: 053602. https://doi.org/10.1103/PhysRevLett.103.053602.

279. Słowik K, Filter R, Straubel J, Lederer F, Rockstuhl C. Strong coupling of optical nanoantennas and atomic systems. Phys Rev B. 2013;88:195414. https://doi.org/10.1103/PhysRevB.88.195414.

280. Kato S, Aoki T. Strong coupling between a trapped single atom and an all-fiber cavity. Phys Rev Lett. 2015;115: 093603. https://doi.org/10.1103/PhysRevLett.115.093603.

281. Esslinger T, Weidemüller M, Hemmerich A, Hänsch TW. Surface-plasmon mirror for atoms. Opt Lett. 1993;18(6): 450-2. https://doi.org/10.1364/OL.18.000450.

282. Chen X, Chen Y-H, Qin J, Zhao D, Ding B, Blaikie RJ, Qiu M. Mode modification of plasmonic gap resonances induced by strong coupling with molecular excitons. Nano Lett. 2017;17(5):3246-51. https://doi.org/10.1021/acs. nanolett.7b00858. PMID: 28394619.

283. Pendry JB, Holden AJ, Stewart WJ, Youngs I. Extremely low frequency plasmons in metallic mesostructures. Phys Rev Lett. 1996;76:4773-6. https://doi.org/10.1103/PhysRevLett.76.4773.

284. Pendry JB, Holden AJ, Robbins DJ, Stewart WJ. Magnetism from conductors and enhanced nonlinear phenomena. IEEE Trans Microw Theory Tech. 1999;47(11):2075-84. https://doi.org/10.1109/22.798002.

285. Smith DR, Pendry JB, Wiltshire MCK. Metamaterials and negative refractive index. Science. 2004;305(5685):788-92. https://doi.org/10.1126/science.1096796.

286. Shalaev VM. Optical negative-index metamaterials. Nat Photonics. 2007;1(1):41-8.

287. Ma Q, Cui TJ. Information metamaterials: bridging the physical world and digital world. PhotoniX. 2020;1 (1):1-32.

288. Zou X, Zheng G, Yuan Q, Zang W, Chen R, Li T, Li L, Wang S, Wang Z, Zhu S. Imaging based on metalenses. PhotoniX. 2020;1(1):1-24.

289. Luo J, Lai Y. Epsilon-near-zero or mu-near-zero materials composed of dielectric photonic crystals. Sci China Inf Sci. 2013;56(12):1-10.

290. Huang X, Lai Y, Hang ZH, Zheng H, Chan C. Dirac cones induced by accidental degeneracy in photonic crystals and zero-refractive-index materials. Nat Mater. 2011;10(8):582-6.

291. Moitra P, Yang Y, Anderson Z, Kravchenko II, Briggs DP, Valentine J. Realization of an all-dielectric zero-index optical metamaterial. Nat Photonics. 2013;7(10):791-5.

292. Mahmoud AM, Engheta N. Wave-matter interactions in epsilon-and-mu-near-zero structures. Nat Commun. 2014;5(1):1-7.

293. Liberal I, Mahmoud AM, Li Y, Edwards B, Engheta N. Photonic doping of epsilon-near-zero media. Science. 2017;355(6329):1058-62. https://doi.org/10.1126/science.aal2672.

294. Veselago VG. The electrodynamics of substances with simultaneously negative values of $\epsilon$ and $\mu$. Sov Phys Usp. 1968;10(4):509-14. https://doi.org/10.1070/pu1968v010n04abeh003699.

295. Sheng C, Liu H, Wang Y, Zhu S, Genov D. Trapping light by mimicking gravitational lensing. Nat Photonics. 2013;7(11):902-6.

296. Tsakmakidis KL, Boardman AD, Hess O. 'trapped rainbow'storage of light in metamaterials. Nature. 2007;450(7168): 397-401.

297. Kildishev AV, Boltasseva A, Shalaev VM. Planar photonics with metasurfaces. Science. 2013;339(6125):. https://doi. org/10.1126/science.1232009.

298. Chen H-T, Taylor AJ, Yu N. A review of metasurfaces: physics and applications. Rep Prog Phys. 2016;79(7):076401.

299. Ding X, Wang Z, Hu G, Liu J, Zhang K, Li H, Ratni B, Burokur SN, Wu Q, Tan J, et al. Metasurface holographic image projection based on mathematical properties of fourier transform. PhotoniX. 2020;1 (1):1-12.

300. Zhao R, Huang L, Wang Y. Recent advances in multi-dimensional metasurfaces holographic technologies. PhotoniX. 2020;1:20.

301. Yu N, Genevet P, Kats MA, Aieta F, Tetienne J-P, Capasso F, Gaburro Z. Light propagation with phase discontinuities: Generalized laws of reflection and refraction. Science. 2011;334(6054):333-7. https://doi.org/10. 1126/science.1210713.

302. Karimi E, Schulz SA, De Leon I, Qassim H, Upham J, Boyd RW. Generating optical orbital angular momentum at visible wavelengths using a plasmonic metasurface. Light: Sci Appl. 2014;3(5):167.

303. Lee J, Tymchenko M, Argyropoulos C, Chen P-Y, Lu F, Demmerle F, Boehm G, Amann M-C, Alu A, Belkin MA. Giant nonlinear response from plasmonic metasurfaces coupled to intersubband transitions. Nature. 2014;511(7507):65-9.

304. Bao Y, Lin Q, Su R, Zhou Z-K, Song J, Li J, Wang X-H. On-demand spin-state manipulation of single-photon emission from quantum dot integrated with metasurface. Sci Adv. 2020;6(31): https://doi.org/10.1126/sciadv. aba8761.

305. Jha PK, Ni X, Wu C, Wang Y, Zhang X. Metasurface-enabled remote quantum interference. Phys Rev Lett. 2015;115:025501. https://doi.org/10.1103/PhysRevLett.115.025501.

306. Staude I, Pertsch T, Kivshar YS. All-dielectric resonant meta-optics lightens up. ACS Photonics. 2019;6(4):802-14.

307. Vaskin A, Kolkowski R, Koenderink AF, Staude I. Light-emitting metasurfaces. Nanophotonics. 2019;8(7):1151-98 https://doi.org/10.1515/nanoph-2019-0110.

308. Shaltout AM, Shalaev VM, Brongersma ML. Spatiotemporal light control with active metasurfaces. Science. 2019;364(6441):eaat3100. https://doi.org/10.1126/science.aat3100.

309. Solntsev AS, Agarwal GS, Kivshar YY. Metasurfaces for quantum photonics. Nat Photonics. 2021;15(5):327-36.

310. Jha PK, Shitrit N, Ren X, Wang Y, Zhang X. Spontaneous exciton valley coherence in transition metal dichalcogenide monolayers interfaced with an anisotropic metasurface. Phys Rev Lett. 2018;121:116102. https:// doi.org/10.1103/PhysRevLett.121.116102.

311. Lassalle E, Lalanne P, Aljunid S, Genevet P, Stout B, Durt T, Wilkowski D. Long-lifetime coherence in a quantum emitter induced by a metasurface. Phys Rev A. 2020;101:013837. https://doi.org/10.1103/PhysRevA.101.013837. 
312. Sohoni $\mathrm{M}$, Jha PK, Nalabothula $\mathrm{M}$, Kumar A. Interlayer exciton valleytronics in bilayer heterostructures interfaced with a phase gradient metasurface. Appl Phys Lett. 2020;117(12):121101. https://doi.org/10.1063/5.0015087.

313. Jha PK, Shitrit N, Kim J, Ren X, Wang Y, Zhang X. Metasurface-mediated quantum entanglement. ACS Photonics. 2017:5(3):971-6.

314. Bucher T, Vaskin A, Mupparapu R, L'ochner FJ, George A, Chong KE, Fasold S, Neumann C, Choi D-Y, Eilenberger $F$, et al. Tailoring photoluminescence from mos 2 monolayers by mie-resonant metasurfaces. ACS Photonics. 2019;6(4):1002-9.

315. Ma X, James AR, Hartmann NF, Baldwin JK, Dominguez J, Sinclair MB, LukTS, Wolf O, Liu S, Doorn SK, et al, Solitary oxygen dopant emission from carbon nanotubes modified by dielectric metasurfaces. ACS Nano. 2017;11(6):6431-9.

316. Lozano G, Louwers DJ, Rodr'iguez SR, Murai S, Jansen OT, Verschuuren MA, Rivas JG. Plasmonics for solid-state lighting enhanced excitation and directional emission of highly efficient light sources. Light Sci Appl. 2013;2(5):66

317. Tanaka K, Plum E, Ou JY, Uchino T, Zheludev NI. Multifold enhancement of quantum dot luminescence in plasmonic metamaterials. Phys Rev Lett. 2010;105:227403. https://doi.org/10.1103/PhysRevLett.105.227403.

318. Tran TT, Wang D, Xu Z-Q, Yang A, Toth M, Odom TW, Aharonovich I. Deterministic coupling of quantum emitters in $2 \mathrm{~d}$ materials to plasmonic nanocavity arrays. Nano Lett. 2017;17(4):2634-9.

319. Yuan S, Qiu X, Cui C, Zhu L, Wang Y, Li Y, Song J, Huang Q, Xia J. Strong photoluminescence enhancement in all-dielectric fano metasurface with high quality factor. ACS Nano. 2017;11(11):10704-11.

320. Cui C, Zhou C, Yuan S, Qiu X, Zhu L, Wang Y, Li Y, Song J, Huang Q, Wang Y, et al. Multiple fano resonances in symmetry-breaking silicon metasurface for manipulating light emission. ACS Photonics. 2018:5(10):4074-80.

321. Liu S, Vaskin A, Addamane S, Leung B, Tsai M-C, Yang Y, Vabishchevich PP, Keeler GA, Wang G, He X, et al. Light-emitting metasurfaces: simultaneous control of spontaneous emission and far-field radiation. Nano Lett. 2018;18(11):6906-14.

322. Chen L, Achouri K, Kallos E, Caloz C. Simultaneous enhancement of light extraction and spontaneous emission using a partially reflecting metasurface cavity. Phys Rev A. 2017;95:053808. https://doi.org/10.1103/PhysRevA.95. 053808.

323. Abass A, Rodriguez SR-K, Ako T, Aubert T, Verschuuren M, Van Thourhout D, Beeckman J, Hens Z, Gómez Rivas J, Maes B. Active liquid crystal tuning of metallic nanoantenna enhanced light emission from colloidal quantum dots. Nano Lett. 2014;14(10):5555-60.

324. Kan Y, Andersen SKH, Ding F, Kumar S, Zhao C, Bozhevolnyi SI. Metasurface-enabled generation of circularly polarized single photons. Adv Mater. 2020;32(16):1907832. https://doi.org/10.1002/adma.201907832.

325. Alam MZ, De Leon I, Boyd RW. Large optical nonlinearity of indium tin oxide in its epsilon-near-zero region. Science. 2016;352(6287):795-7. https://doi.org/10.1126/science.aae0330.

326. Maas R, Parsons J, Engheta N, Polman A. Experimental realization of an epsilon-near-zero metamaterial at visible wavelengths. Nat Photonics. 2013;7(11):907-12.

327. Caldwell JD, Lindsay L, Giannini V, Vurgaftman I, Reinecke TL, Maier SA, Glembocki OJ. Low-loss, infrared and terahertz nanophotonics using surface phonon polaritons. Nanophotonics. 2015;4(1):44-68.

328. Huang $X$, Lai $Y$, Hang $Z H$, Zheng $H$, Chan C. Dirac cones induced by accidental degeneracy in photonic crystals and zero-refractive-index materials. Nat Mater. 2011;10(8):582-6.

329. Vassant S, Archambault A, Marquier F, Pardo F, Gennser U, Cavanna A, Pelouard JL, Greffet JJ. Epsilon-near-zero mode for active optoelectronic devices. Phys Rev Lett. 2012;109:237401. https://doi.org/10.1103/PhysRevLett.109. 237401.

330. Kim J, Dutta A, Naik GV, Giles AJ, Bezares FJ, Ellis CT, Tischler JG, Mahmoud AM, Caglayan H, Glembocki OJ, Kildishev AV, Caldwell JD, Boltasseva A, Engheta N. Role of epsilon-near-zero substrates in the optical response of plasmonic antennas. Optica. 2016:3(3):339-46. https://doi.org/10.1364/OPTICA.3.000339.

331. Ou J-Y, So J-K, Adamo G, Sulaev A, Wang L, Zheludev NI. Ultraviolet and visible range plasmonics in the topological insulator bi 1.5 sb 0.5 te 1.8 se 1.2. Nat Commun. 2014;5(1):1-7.

332. Anderegg M, Feuerbacher B, Fitton B. Optically excited longitudinal plasmons in potassium. Phys Rev Lett. 1971;27:1565-8. https://doi.org/10.1103/PhysRevLett.27.1565.

333. Vesseur EJR, Coenen T, Caglayan H, Engheta N, Polman A. Experimental verification of $n=0$ structures for visible light. Phys Rev Lett. 2013;110:013902. https://doi.org/10.1103/PhysRevLett.110.013902.

334. Silveirinha M, Engheta N. Tunneling of electromagnetic energy through subwavelength channels and bends using $\epsilon$-near-zero materials. Phys Rev Lett. 2006;97:157403. https://doi.org/10.1103/PhysRevLett.97.157403.

335. Silveirinha MG, Engheta N. Theory of supercoupling, squeezing wave energy, and field confinement in narrow channels and tight bends using $\epsilon$ near-zero metamaterials. Phys Rev B. 2007;76:245109. https://doi.org/10.1103/ PhysRevB.76.245109.

336. Edwards B, Alù A, Young ME, Silveirinha M, Engheta N. Experimental verification of epsilon-near-zero metamaterial coupling and energy squeezing using a microwave waveguide. Phys Rev Lett. 2008;100:033903. https://doi.org/10.1103/PhysRevLett.100.033903.

337. Xu J, Song G, Zhang Z, Yang Y, Chen H, Zubairy MS, Zhu S. Unidirectional single-photon generation via matched zero-index metamaterials. Phys Rev B. 2016;94:220103. https://doi.org/10.1103/PhysRevB.94.220103.

338. Enoch S, Tayeb G, Sabouroux P, Guérin N, Vincent P. A metamaterial for directive emission. Phys Rev Lett. 2002;89:213902. https://doi.org/10.1103/PhysRevLett.89.213902.

339. Suchowski H, O'Brien K, Wong ZJ, Salandrino A, Yin X, Zhang X. Phase mismatch-free nonlinear propagation in optical zero-index materials. Science. 2013;342(6163):1223-6. https://doi.org/10.1126/science.1244303.

340. Alam MZ, De Leon I, Boyd RW. Large optical nonlinearity of indium tin oxide in its epsilon-near-zero region. Science. 2016;352(6287):795-7. https://doi.org/10.1126/science.aae0330. https://science.sciencemag.org/content/ 352/6287/795.full.pdf.

341. Neira AD, Olivier N, Nasir ME, Dickson W, Wurtz GA, Zayats AV. Eliminating material constraints for nonlinearity with plasmonic metamaterials. Nat Commun. 2015;6(1):1-8.

342. Caspani L, Kaipurath RPM, Clerici M, Ferrera M, Roger T, Kim J, Kinsey N, Pietrzyk M, Di Falco A, Shalaev VM, Boltasseva A, Faccio D. Enhanced nonlinear refractive index in $\epsilon$-near-zero materials. Phys Rev Lett. 2016;1 16: 233901. https://doi.org/10.1103/PhysRevLett.116.233901. 
343. Zhao Y, Yang Y, Sun H-B. Nonlinear meta-optics towards applications. PhotoniX. 2021;2(3):1.

344. Engheta N, Salandrino A, Alù A. Circuit elements at optical frequencies: Nanoinductors, nanocapacitors, and nanoresistors. Phys Rev Lett. 2005;95:095504. https://doi.org/10.1103/PhysRevLett.95.095504.

345. Engheta N. Circuits with light at nanoscales: Optical nanocircuits inspired by metamaterials. Science. 2007;317(5845):1698-702. https://doi.org/10.1126/science.1133268. https://science.sciencemag.org/content/317/ 5845/1698.full.pdf.

346. Alù A, Engheta N. All optical metamaterial circuit board at the nanoscale. Phys Rev Lett. 2009;103:143902. https:// doi.org/10.1103/PhysRevLett.103.143902.

347. Schulz SA, Tahir AA, Alam MZ, Upham J, De Leon I, Boyd RW. Optical response of dipole antennas on an epsilon-near-zero substrate. Phys Rev A. 2016;93:063846. https://doi.org/10.1103/PhysRevA.93.063846.

348. Liberal I, Engheta N. Decay dynamics of quantum emitters in epsilon-near-zero cavities. In: 2016 Conference on Lasers and Electro-Optics (CLEO). San Jose: IEEE; 2016. p. 1-2.

349. Liberal I, Mahmoud AM, Engheta N. Geometry-invariant resonant cavities. Nat Commun. 2016;7(1):1-7.

350. Alù A, Engheta N. Boosting molecular fluorescence with a plasmonic nanolauncher. Phys Rev Lett. 2009;103: 043902. https://doi.org/10.1103/PhysRevLett.103.043902.

351. Campione S, de Ceglia D, Vincenti MA, Scalora M, Capolino F. Electric field enhancement in $\varepsilon$-near-zero slabs under tm-polarized oblique incidence. Phys Rev B. 2013;87:035120. https://doi.org/10.1103/PhysRevB.87.035120.

352. Sokhoyan R, Atwater HA. Quantum optical properties of a dipole emitter coupled to an $\epsilon$-near-zero nanoscale waveguide. Opt Express. 2013;21(26):32279-90. https://doi.org/10.1364/OE.21.032279.

353. Fleury R, Alù A. Enhanced superradiance in epsilon-near-zero plasmonic channels. Phys Rev B. 2013;87:201101. https://doi.org/10.1103/PhysRevB.87.201101.

354. Liberal I, Engheta N. Nonradiating and radiating modes excited by quantum emitters in open epsilon-near-zero cavities. Sci Adv. 2016;2(10):. https://doi.org/10.1126/sciadv.1600987.

355. Campione S, Marquier F, Hugonin J-P, Ellis AR, Klem JF, Sinclair MB, Luk TS. Directional and monochromatic thermal emitter from epsilon-near-zero conditions in semiconductor hyperbolic metamaterials. Sci Rep. 2016;6(1): $1-9$.

356. Kamandar Dezfouli M, Gordon R, Hughes S. Modal theory of modified spontaneous emission of a quantum emitter in a hybrid plasmonic photonic-crystal cavity system. Phys Rev A. 2017;95:013846. https://doi.org/10.1103/ PhysRevA.95.013846.

357. Xiao M, Zhang ZQ, Chan CT. Surface impedance and bulk band geometric phases in one-dimensional systems. Phys Rev X. 2014;4:021017. https://doi.org/10.1103/PhysRevX.4.021017.

358. Lu L, Joannopoulos JD, Soljačić M. Topological photonics. Nat Photonics. 2014;8(11):821-9.

359. Khanikaev AB, Shvets G. Two-dimensional topological photonics. Nat Photonics. 2017;11(12):763-73.

360. Tiecke T, Thompson JD, de Leon NP, Liu L, Vuletić V, Lukin MD. Nanophotonic quantum phase switch with a single atom. Nature. 2014;508(7495):241-4.

361. McKeever J, Boca A, Boozer AD, Buck JR, Kimble HJ. Experimental realization of a one-atom laser in the regime of strong coupling. Nature. 2003;425(6955):268-71.

362. Blanco-Redondo A, Bell B, Oren D, Eggleton BJ, Segev M. Topological protection of biphoton states. Science. 2018;362(6414):568-71. https://doi.org/10.1126/science.aau4296. https://science.sciencemag.org/content/362/ 6414/568.full.pdf.

363. Wang Y, Lu Y-H, Mei F, Gao J, Li Z-M, Tang H, Zhu S-L, Jia S, Jin X-M. Direct observation of topology from single-photon dynamics. Phys Rev Lett. 2019;122:193903. https://doi.org/10.1103/PhysRevLett.122.193903.

364. Mittal S, Goldschmidt EA, Hafezi M. A topological source of quantum light. Nature. 2018;561 (7724):502-6.

365. Xie X, Zhang W, He X, Wu S, Dang J, Peng K, Song F, Yang L, Ni H, Niu Z, Wang C, Jin K, Zhang X, Xu X. Cavity quantum electrodynamics with second-order topological corner state. Laser Photonics Rev. 2020;14(8):1900425. https://doi.org/10.1002/lpor.201900425.

366. Miri M-A, Alù A. Exceptional points in optics and photonics. Science. 2019;363(6422):eaar7709. https://doi.org/10 1126/science.aar7709.

367. Turner MD, Saba M, Zhang Q, Cumming BP, Schröder-Turk GE, Gu M. Miniature chiral beamsplitter based on gyroid photonic crystals. Nat Photonics. 2013;7(10):801-5.

368. Defienne H, Ndagano B, Lyons A, Faccio D. Polarization entanglement-enabled quantum holography. Nat Phys. 2021;17:591-7.

369. Bhaskar MK, Riedinger R, Machielse B, Levonian DS, Nguyen CT, Knall EN, Park H, Englund D, Lončar M, Sukachev DD, et al. Experimental demonstration of memory-enhanced quantum communication. Nature. 2020;580(7801):60-4.

\section{Publisher's Note}

Springer Nature remains neutral with regard to jurisdictional claims in published maps and institutional affiliations. 